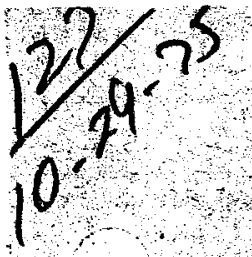

$e n-171$

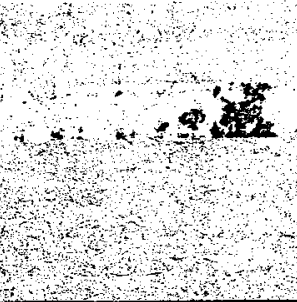

잘

\title{
VAPOR-DROPLET FLOW EQUATIONS
}

\author{
C. T. Crowe
}

August 18, 1975

Prepared for U.S. Energy Research \& Development Administration under contract No. W-7405-Eng- 48

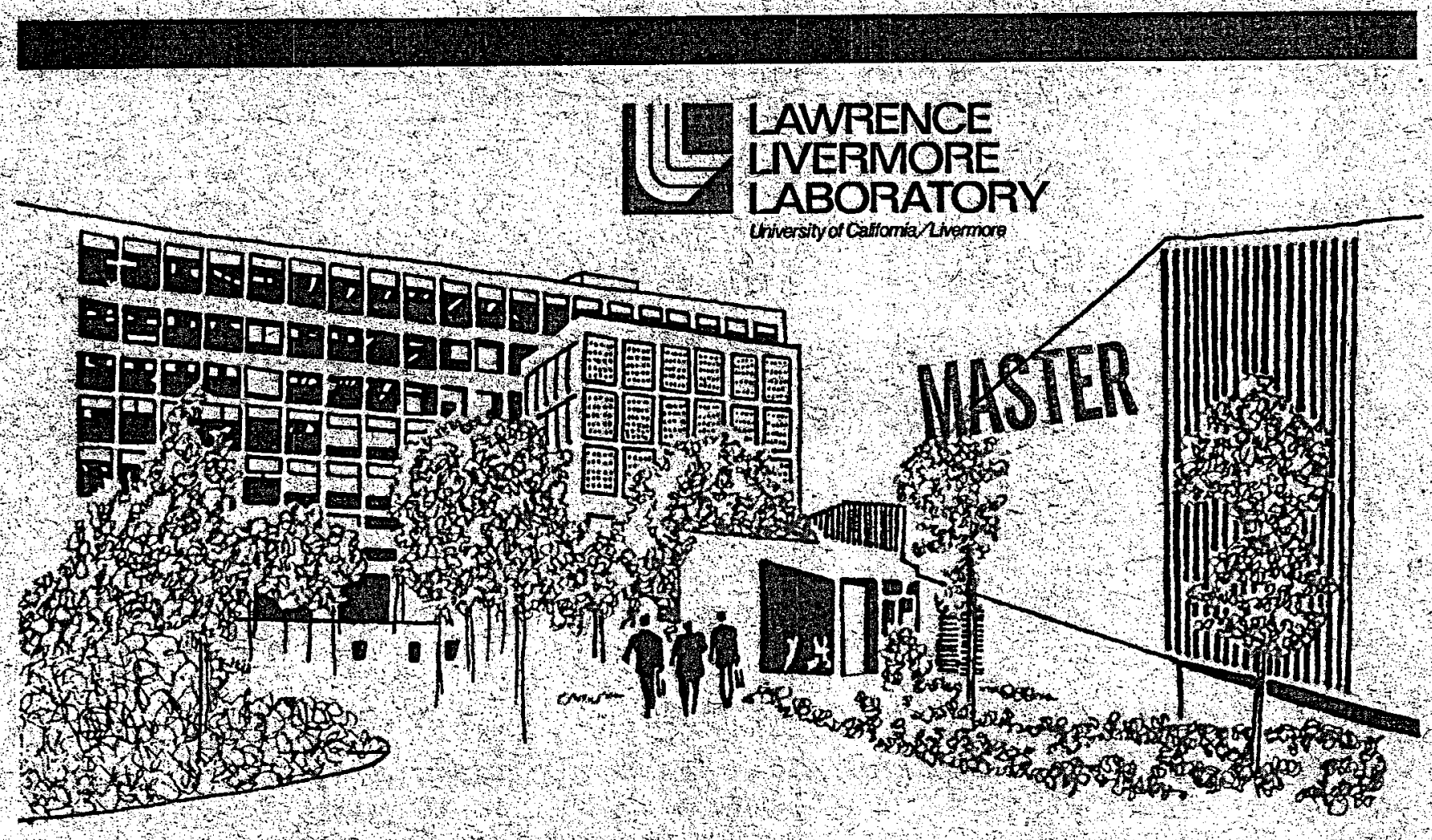




\section{DISCLAIMER}

This report was prepared as an account of work sponsored by an agency of the United States Government. Neither the United States Government nor any agency Thereof, nor any of their employees, makes any warranty, express or implied, or assumes any legal liability or responsibility for the accuracy, completeness, or usefulness of any information, apparatus, product, or process disclosed, or represents that its use would not infringe privately owned rights. Reference herein to any specific commercial product, process, or service by trade name, trademark, manufacturer, or otherwise does not necessarily constitute or imply its endorsement, recommendation, or favoring by the United States Government or any agency thereof. The views and opinions of authors expressed herein do not necessarily state or reflect those of the United States Government or any agency thereof. 


\section{DISCLAIMER}

Portions of this document may be illegible in electronic image products. Images are produced from the best available original document. 


\title{
㢟 \\ LAWRENCE LNERMORE LABORATORY \\ University of Caffornia/Livermore, California/ 94550
}

\section{UCR L-51877 \\ VAPOR-DROPLET FLOW EQUATIONS}

\author{
C. T. Crowe
}

MS. date: August 18, 1975

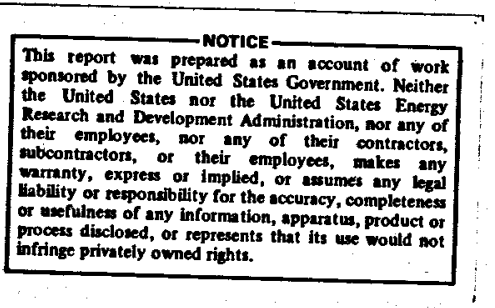


C 


\section{Contents}

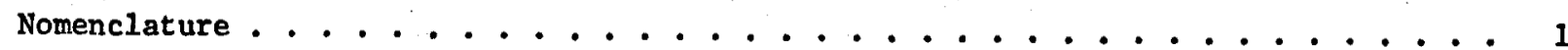

Greek Letters .......................... 2

Abstract . . . . . . . . . . . . . . . . . . . . . 2

Introduction ........................... 3

Transport theorem ........................... . . 4

Lagranglan Equation for a Droplet . . . . . . . . . . . . . . . 5

Continulty Equation . . . . . . . . . . . . . . . . . 6

Momentum Equation . . . . . . . . . . . . . . . . 6

Energy Equation .......................... 8

Eulerian Equations for Droplet Cloud . . . . . . . . . . . . . 10

Continulty Equation......................... 11

Momentum Equation . . . . . . . . . . . . . . . . . . . 12

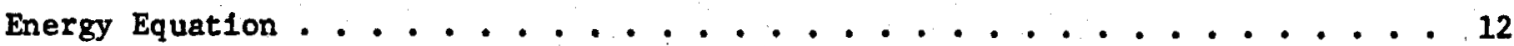

Eulertan Equations for Vapor Phase . . . . . . . . . . . . . . . . . 14

Continuity Equation . . . . . . . . . . . . . . . . . 14

Momentum Equation . . . . . . . . . . . . . . . . . 15

Energy Equation ........................ 16

Mixture Equations. ......................... 19

Continufty Equations . . . . . . . . . . . . . . . 19

Momentum Equation . . . . . . . . . . . . . . . . . 19

Energy Equation . . . . . . . . . . . . . . . . . . 19

Interphase Property Transfer . . . . . . . . . . . . . . . . . . 20

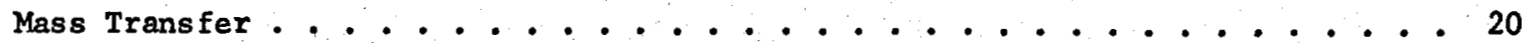

Momentum Transfer . . . . . . . . . . . . . . . . . 23

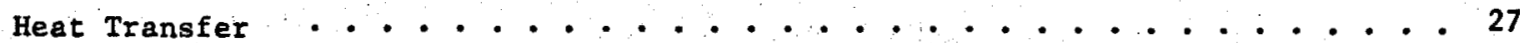

Acknowledgments. . . . . . . . . . . . . . . . . . . . 28

References . . . . . . . . . . . . . . . . . . . . 29 


\section{Nomenclature}

\begin{tabular}{|c|c|c|c|}
\hline A & area & $\ell *$ & length scale \\
\hline $\mathrm{A}_{\mathrm{c}}$ & acceleration modulus & m & mass of droplet \\
\hline b & Intensive property of a system & $\dot{\mathrm{m}}$ & mass flow rate \\
\hline B & extensive property of a system & $\operatorname{Mom}_{1}$ & momentum vector \\
\hline$c_{A}$ & concentration of specie $A$ & M & mass of system \\
\hline$c_{d}$ & spectflc heat of droplet & $\mathrm{n}_{1}$ & unit outward normal vector \\
\hline$c_{8}$ & concentration at surface & N & number of droplets per unft \\
\hline$c_{\infty}$ & concentration far from surface & & volume \\
\hline$C_{D}$ & drag coefficient & p & pressure \\
\hline$d$ & droplet diameter & P.E. & potential energy \\
\hline D & diffusion coefficlent & $\dot{q}_{d}$ & heat transfer rate to droplets per \\
\hline e & spectfic energy & & unit volume of mixture \\
\hline $\mathbf{E}$ & energy & $\dot{q}_{1}$ & heat transfer rate per unft area \\
\hline$f_{d, 1}$ & aerodynamic force on droplets per & & (vector) \\
\hline & unit volume of mixture & $\dot{Q}$ & heat transfer rate \\
\hline$F_{1}$ & force vector & $\dot{\mathrm{Q}}_{\mathrm{d}}$ & heat transfer rate to droplet \\
\hline$F_{B, 1}$ & Basset force vector & $r_{1}$ & radius vector \\
\hline$F_{d, 1}$ & aerodynamic force on droplet & $r_{\mathrm{d}, 1}$ & radius vector a droplet surface \\
\hline$F_{D, 1}$ & aerodynamic drag on droplet & $r_{d}$ & $\begin{array}{l}\text { radius of spherical droplet } \\
\text { Revnolds number }\end{array}$ \\
\hline$F_{p, 1}^{m, 1}$ & buoyancy force & $s_{m}$ & evaporation rate of droplets per \\
\hline$F_{r, 1}$ & Magnus force vector & & untt volume of mixture \\
\hline $\mathrm{F}_{\mathrm{S}}$ & Saffman lift force & $\mathbf{s}$ & control surface \\
\hline & enthalpy of vapor phase & $s_{m}$ & evaporation rate of droplets \\
\hline${ }^{h}$ fg & $\begin{array}{l}\text { enthalpy difference between vapor } \\
\text { and liquid }\end{array}$ & $s_{p}$ & $\begin{array}{l}\text { control surface enclosing interior } \\
\text { droplets }\end{array}$ \\
\hline$h_{s}$ & $\begin{array}{l}\text { enthalpy of vapor at droplet } \\
\text { surface }\end{array}$ & $S_{p}$ & $\begin{array}{l}\text { control surface enclosing interior } \\
\text { and "inside-boundary" droplets }\end{array}$ \\
\hline $1_{d}$ & $\begin{array}{l}\text { speciftc internal energy of } \\
\text { droplet }\end{array}$ & $\partial S$ & $\begin{array}{l}\text { portion of } S \text { intercepted by } \\
\text { boundary droplets }\end{array}$ \\
\hline$I_{\mathrm{d}}$ & Internal energy of droplet & $\mathrm{s}_{\mathrm{p}}$ & $\begin{array}{l}\text { control surface on interior side } \\
\text { of boundary droplets }\end{array}$ \\
\hline $\begin{array}{l}\text { t } \\
k\end{array}$ & thermal conductivity & Sh & Sherwood number \\
\hline $\mathrm{K}_{\mathrm{B}}$ & coefficlent for Basset force & $\begin{array}{l}\text { Sc } \\
t\end{array}$ & $\begin{array}{l}\text { Schmidt number } \\
\text { time }\end{array}$ \\
\hline K.E. & kinetic energy & $t *$ & time scale \\
\hline $\begin{array}{l}k_{m} \\
k n\end{array}$ & $\begin{array}{l}\text { coefficient for virtual mass } \\
\text { Knudsen number }\end{array}$ & $t_{d}$ & $\begin{array}{l}\text { characteristic time for droplet } \\
\text { heat transfer }\end{array}$ \\
\hline
\end{tabular}




$\begin{array}{ll}t_{p} & \begin{array}{l}\text { characteristic time for pressure } \\ \text { change }\end{array} \\ T_{d} & \text { droplet temperature } \\ T_{v} & \text { vapor temperature } \\ u & \text { magnitude of velocity } u_{1} \\ u_{i} & \text { droplet velocity with respect to } \\ & \text { an inertial reference frame } \\ u_{*} & \text { shear velocity in boundary layer } \\ v & \text { velocity with respect to an } \\ v_{1} & \text { inertial reference frame } \\ v & \text { vapor velocity } \\ v_{p} & \text { volume of individual droplet } \\ v_{d} & \text { volume occupied by droplets } \\ v_{v} & \text { volume occupled by vapor } \\ w & \text { magnitude of velocity w }\end{array}$

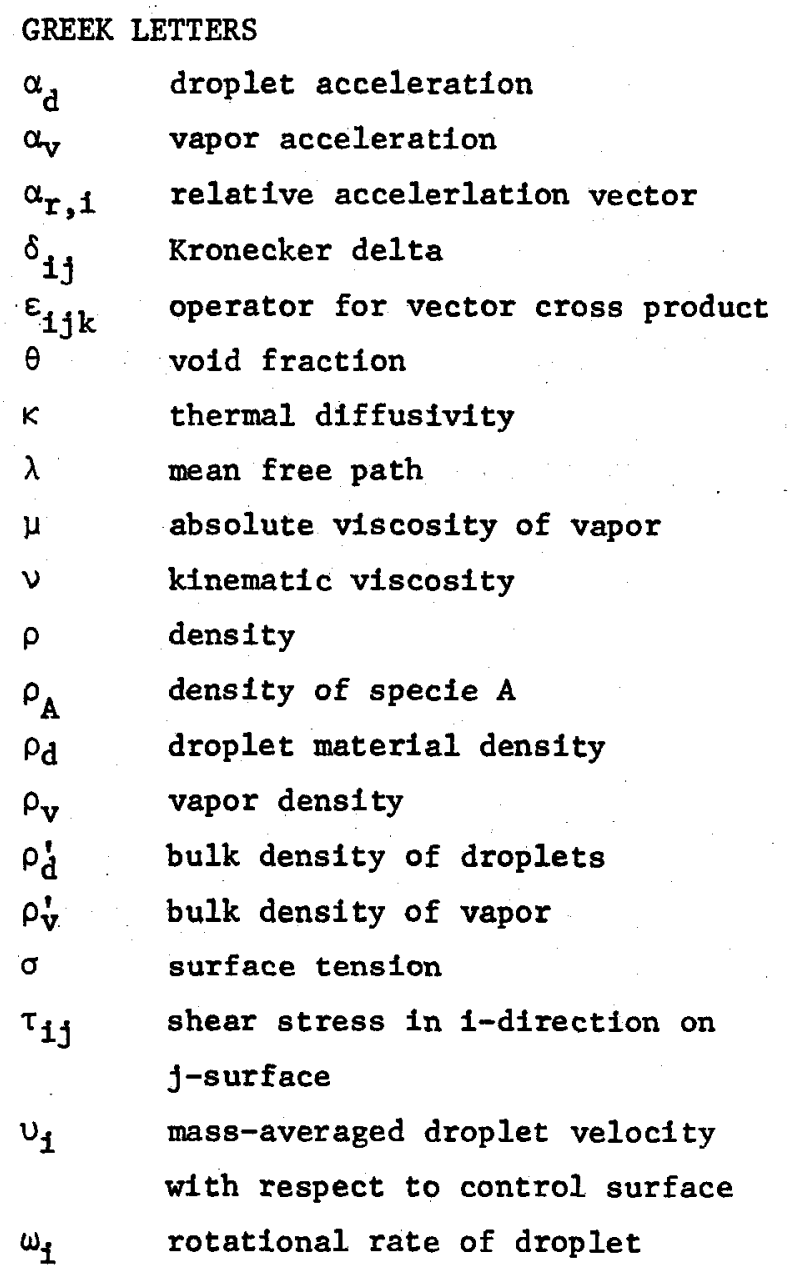

\title{
VAPOR-DROPLET FLOW EQUATIONS
}

\begin{abstract}
This report discusses the general features of a vapor-droplet flow and derives the equations expressing the conservation of mass, momentum, and energy for the vapor, 1iquid, and mixture using the control volume approach.

The phenomenological laws describing the exchange of mass, momentum, and energy between phases are also reviewed. The results have application to development of water-dominated geothermal
resources.
\end{abstract}




\section{Introduction}

Equations describing the conservation of mass, momentum, and energy of a flowing vapor-droplet suspension can be found in several books, ${ }^{1,2}$ papers, ${ }^{3,4}$ and reports. In some references, the equations are incorrect; in others, they are presented In a form too complex to be of utility to the practicing englneer. Very few analytic solutions for two-phase flows can be found in the literature owing to the complexity of the equations. Numerous numerical solutions for one-dimensional (and quasi one-dimensional) flows 5,6 have appeared, but numerical solutions for two- and three-dimensional two-phase flows are scarce and, in some cases, the governIng equations used are incorrect. The purpose of this report is to present a careful, stralghtforward derivation of the governing equations from which valid numerical solutions schemes can be developed.

The most significant feature of a vapor-droplet flow is the fact that the droplets do not constitute a continuum. Thus, strictly speaking, one cannot define droplet density at a point in the flow. The only meaningful definftion of droplet density is bulk density or mass of droplets per unit volume of mixture. Thus, the mixture volume whlch one conslders must contain many droplets in order to define an "average." Yet, if differential equations are to be used to express property conservation, the volume must be small enough to be considered a mathematical "point." The same argument applies to the definition of droplet velocity and temperature.
Drew $^{7}$ has published a very thorough, though complicated, derivation of the differential form of the governing equations for a two-phase flow. He defines average properties based on length and tIme scales $l^{*}$ and $t *$, which are the dimensions of a space and time volume fust sufficient to establish a "stationary" average; that is, the average will not change by taking even larger values for $\ell$ * and $t *$. He also takes an "average" of the averages in the same fashion to ensure continuity of the space and time derivatives. He implies that the error in the predicted average properties is the sum of the orders of $\ell^{*}$ and $t *$. Thus, if $\ell *$ or t* are large with reference to the size or the characterlstic dimension of the flow system, then the differential equations of continua are inadequate to describe the motion of the dispersed phase.

The validity of differential equations In describing a vapor-droplet flow can be crudely assessed by assuming that $l *$ should be of the order of $10^{-2}$ (for about $1 \%$ accuracy) and that a spatial volume should contain at least $10^{3}$ droplets to obtain a stationary average. For example, consider the flow through a 64-in. ${ }^{3}$ volume (a cube with 4-in. sides). Assume the vapor has a denstty of 0.01 pounds mass $/ \mathrm{ft}^{3}\left(1 \mathrm{bm} / \mathrm{ft}^{3}\right)$, that the droplets are water $5 \mu \mathrm{m}$ in diameter, and that the quality of the mixture is $50 \%$. The number density of droplets is approximately $10^{11}$ per cubic foot. Taking $l * \sim 10^{-2}$, then the volume over which the average must be stationary is $64 \times 10^{-6}$ in. $^{3}$ or $3.7 \times 10^{-8} \mathrm{ft}^{3}$ This 
volume would contain somewhat more than $10^{3}$ droplets and would probably suffice to define a stationary average. Thus, in this example, the average values obtained by solving the differential equations will probably not be more than $1 \%$ accurate, if that.

This report derives the governing equations for vapor-droplet flow. The equations are presented in both integral and differential forms. No attempt is made to define average properties according to Drew. 7 Equations expressing the mass, momentum, and energy conservation for the single droplet are derived first and utilized in the equations for a droplet cloud. The vapor-phase equations are then derfved and added to the dropletcloud equations to yield the mixture equations. Finally, the phenomenological laws pertaining to transfer of mass. momentum, and energy between phases are reviewed and discussed.

To avoid the unwieldiness of the equations, index notation is used throughout this report. The component of vector $\vec{v}$ in the 1 -direction is simply $v_{1}$. The dot product $\vec{v} \cdot \vec{n}$ is expressed as

$$
\overrightarrow{\mathbf{v}} \cdot \overrightarrow{\mathbf{n}}=\mathbf{v}_{\alpha} \mathrm{n}_{\alpha},
$$

which implies sumation over double

indices, i.e.,

$$
\begin{aligned}
\vec{v} \cdot \vec{n} & =v_{\alpha} n_{\alpha} \\
& =v_{1} n_{1}+v_{2} n_{2}+v_{3} n_{3}
\end{aligned}
$$

For convenfence and clarity, Greek subscrlpts are used to indicate summed indices and Latin subscripts are used to indicate component direction. For example, a corollary of the divergence theorem used in the momentum equation is

$$
\begin{aligned}
& \int_{S}\left(v_{\alpha} n_{\alpha} v_{1}\right) d A= \\
& \int_{v} \frac{\partial}{\partial x_{\alpha}}\left(v_{\alpha} v_{1}\right) d v
\end{aligned}
$$

which gives the component in the $i-t h$ direction. Using index notation allows one to recognize the essence of the equations unencumbered by mathematical mantpulations. Fundamental to the derivation of the equations is the transport theorem which, for completeness, is reviewed in the following section.

\section{Transport Theorem}

Consider an identifiable portion of matter (a system) which consists always of the same matter and moves with time through space. This portion of mass may, or may not, contain both liquid and vapor phases. The system has extensive properties such as momentum and energy which may vary with time. Expressing the conservation laws for the system's properties as they vary in time constitutes the Lagrangian description of the flowing medium.

Although the Lagrangian approach is the most convenient for mechanical systems, the motion of a moving fluid is best analyzed using the Eulerian approach in which conservation equations are written for the flow of properties through a 
(a)

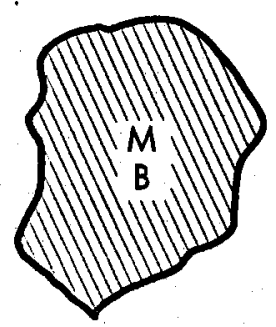

(b)

Control volume-

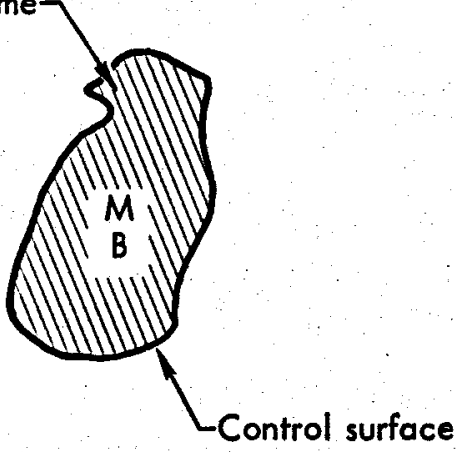

F1g. 1. System, control surface, and control volume: (a) intial conditions, (b) after the system has flowed through and just occupied the control volume.

volume defined in space (a control volume). The theorem which relates the change of a property of a system with time to the flow through and changes within a control volume is the transport theorem.
Consider a mass $M$ which moves through space and with $B$ as some extensive property of that mass. Figure la shows mass $M$ and a control volume separated in space. After some time, the system moves through and just occupies the control volume, as shown in Fig. 1b. At this Instant, the transport theorem states that

$\frac{d B}{d t}=\frac{d}{d t} \int_{c . v .} b \rho d v+\int_{c . s .} \rho b v_{\alpha}{ }^{n} \alpha^{d A}$,

where $b$ is the corresponding intensive property, and c.v. and c.s. represent the control volume and control surface, respectively. The vector $v_{1}$ represents the velocity of the mass flowing across the control surface and is referenced with respect to the control surface. The vector $n_{1}$ is the unit outward normal vector. In words, the transport theorem states that the rate of change of property $B$ of a system is equal to the rate of change of property $B$ within the control volume plus the net efflux of property B through the control surface at the Instant the system occupies the control volume. The control volume may also move through space with time. This equation 18 derived in most fluid mechanlcs texts. It is imperative that the essentials of the theorem be understood In its application to a dropletvapor flow.

\section{Lagrangian Equations for a Droplet}

The transport theorem 1s now applied to the conservation of mass, momentum, and energy of a droplet. Constder the droplet shown in Fig. 2. The control volume moves with the droplet and the control surface is outside of and adjacent to the droplet-vapor interface. The velocity $w_{1}$ is defined as the velocity of vapor through the control surface with respect to the control surface. The 


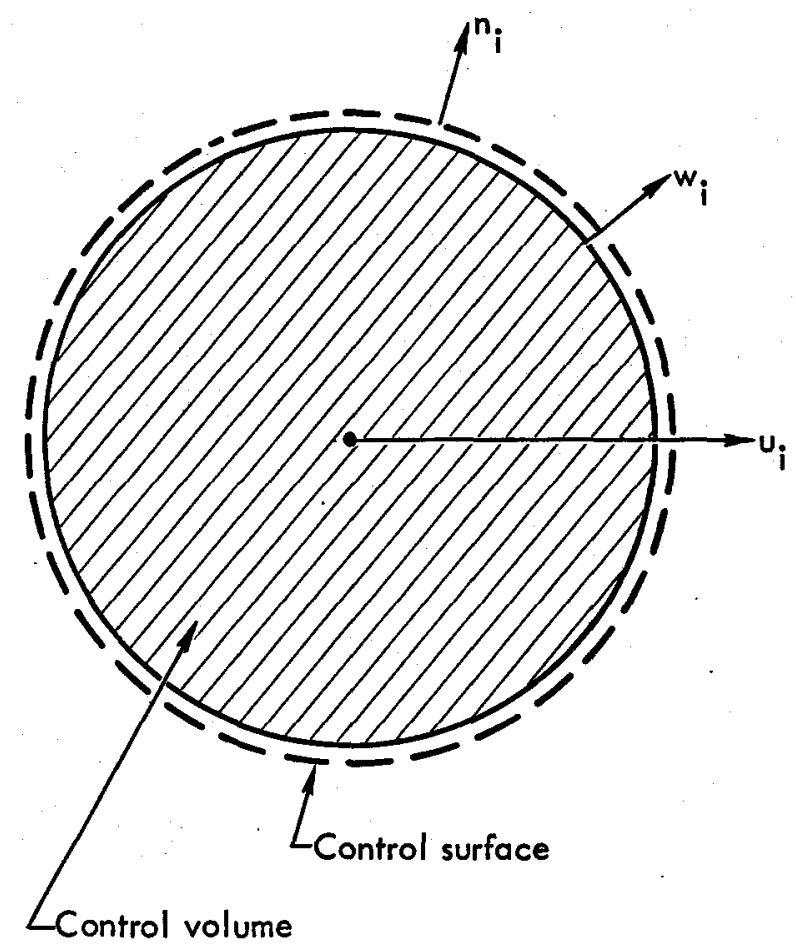

Fig. 2. Control surface and volume for moving droplet.

density of the liquid phase is $\rho_{d}$ (assumed constant throughout droplet) and the density of the vapor at the control surface is $\rho_{\mathrm{v}}$.

CONTINUITY EQUATION

By definition, the mass of a system of fluid particles in Invariant, and the intensive property corresponding to mass (mass per unit mass) is unity. Thus the transport theorem reduces to

$$
\begin{aligned}
\frac{d M}{d t}=0= & \frac{d}{d t} \int_{c . v \cdot} \rho_{d} d V \\
& +\int_{c . s .} \rho_{v}{ }^{w} \alpha^{n} \alpha d A
\end{aligned}
$$

However, by definition,

$$
\int_{c . v .} \rho_{d} d V=m
$$

which is the mass of the droplet, so

$$
\int_{\text {. s. }} \rho_{v}{ }^{w}{ }_{\alpha}{ }_{\alpha} d A=-\frac{d m}{d t}
$$

This equation simply states that the net efflux of mass from the control surface is equal to the rate of mass decrease of the droplet. If the mass flux is constant over the droplet surface, then

$\int_{c .8 .} \rho_{v} w_{\alpha}{ }^{n} d A=\rho_{v} w A=-\frac{d m}{d t}$,

where A is the surface area of the droplet.

\section{MOMENTUM EQUATION}

Assume the droplet is moving at a velocity $u_{1}$ with respect to an inertial reference frame and rotating as a solid body about its center of mass with a rotation vector $\omega_{1}$. The velocity at any point within the droplet with respect to the inertial reference frame is*

$$
u_{1}+\varepsilon_{i \alpha \beta} \omega_{\alpha \beta} r_{\beta},
$$

and the velocity at the surface of the droplet is ${ }^{\dagger}$

$$
u_{1}+\varepsilon_{1 \alpha \beta} w_{\alpha} r_{\beta, d}+w_{1} \text {, }
$$

\footnotetext{
* $\varepsilon_{i j k}$ is the operator corresponding to the vector product. It has a magnitude of unity and its sign depends on the order of $1, j$, and $k$.

†Actually; the droplet surface moves as evaporation or condensation occur. However, this velocity is equal to the vapor-liquid density ratio times $w$ and can usually be neglected compared to $w$.
} 
where $r_{1, d}$ is the radius vector at the droplet surface.

Newton's second law states that the forces acting on a group of fluid particles (a system) is equal to the net rate of change of momentum of the system:

$$
F_{1}=\frac{d}{d t}\left(\operatorname{Mom}_{1}\right)
$$

Momentum is an extensive property of the system and the corresponding intensive property, momentum per unit mass, is the velocity with respect to an Inertial reference frame. The transport theorem for the droplet can be written as

$$
\begin{aligned}
F_{1}= & \frac{d}{d t} \int_{c . v .} \rho_{d}\left(u_{1}+\varepsilon_{1 \alpha \beta} w_{\alpha} r_{\beta}\right) d V \\
& +\int_{c . s .}\left(u_{1}+\varepsilon_{1 \alpha \beta} w_{\alpha} r_{B, d}+w_{1}\right) \\
& \rho_{v}{ }^{w_{\gamma} n_{\gamma} d A},
\end{aligned}
$$

where the Force $F_{1}$ constitutes the force acting on the droplet. Because $u_{1}$ and $w_{1}$ are constant throughout the droplet, the first integral can be written as

$$
\begin{aligned}
\int c . v . & \rho_{d}\left(u_{1}+\varepsilon_{I \alpha \beta} w_{\alpha} r_{\beta}\right) d V=u_{1} \int_{c . v .} \rho_{d} d V \\
& +\varepsilon_{1 \alpha \beta}{ }^{\omega_{\alpha}} \int_{c . v .} \rho_{d} r_{\beta} d v
\end{aligned}
$$

However,

$$
\int_{c . v_{.}} \rho_{d} d V=m
$$

and by definition of the center of mass

$$
\int_{c . v .} \rho_{D} r_{1} d V=0
$$

so

$$
\begin{aligned}
& \frac{d}{d t} \int_{c, v .} \rho_{d}\left(u_{i}+\varepsilon_{i \alpha \beta} \omega_{\alpha \beta} r_{\beta} d V\right. \\
& =\frac{d}{d t}\left(m u_{i}\right)=u_{i} \frac{d m}{d t}+m \frac{d u_{1}}{d t} .
\end{aligned}
$$

The second term, or efflux term, of Eq. (10), can be expanded to

$$
\begin{aligned}
& \int_{c, 8 .}\left(u_{1}+\varepsilon_{1 \alpha \beta} w_{\alpha} r_{\beta, d}+w_{1}\right) p_{v} w_{\gamma}^{n_{\gamma}} d A \\
& =u_{1} \int_{c .8 .} \rho_{v} w_{\alpha} \alpha^{d A} \\
& +\varepsilon_{1 \alpha \beta}{ }^{w} \int_{c .8 .} r_{\beta, d^{\rho}}{ }^{w}{ }^{w}{ }^{n} \alpha A
\end{aligned}
$$

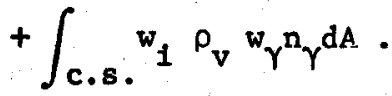

Using the continuity equation, Eq. (8), the first term becomes

$$
u_{1} \int_{c . s .} \rho_{v} w_{\alpha} n_{\alpha} d A=-u_{1} \frac{d m}{d t}
$$

If the mass flux velocity is constant over the surface of the droplet, then

$$
w_{1}=w_{1}
$$

so

$$
\rho_{v}{ }^{n} \alpha^{n} d A=\rho_{v} w d A
$$

and the third term reduces to zero

$$
\begin{aligned}
& \int_{\text {c.s. }} w_{1}^{p_{v} w_{\alpha}{ }^{n} d A} \\
& =\rho_{v} w^{2} \int_{c . s .} n_{1} d A=0
\end{aligned}
$$


If, In addition, the droplet is spherical with radius $r_{d}$, the second term also becomes zero,

$$
\begin{aligned}
& \int_{c . s .} r_{i, d} \rho_{v}{ }^{w}{ }^{n}{ }_{\alpha}^{d A} \\
& =r_{d} \rho_{v} w \int_{c . s .} n_{i} d A=0 .
\end{aligned}
$$

If the droplet is not exchanging mass uniformly over its surface, the second and third terms may not be zero but are generally negligible which can be shown by an order of magnitude analysis.

Finally, collecting all terms and substituting them into the transport theorem yields

$$
F_{i}=m \frac{d u_{i}}{d t} .
$$

Thus, the force acting on a spherical, rotating droplet is equal to the product of its instantaneous mass and the acceleration of 1 ts center of mass.

often, in the literature, one sees Newton's second law written as

$$
F_{i}=\frac{d}{d t}\left(m u_{i}\right)
$$

and differentiated to give

$$
F_{i}=m \frac{d u_{i}}{d t}+u_{i} \frac{d m}{d t} .
$$

This is incorrect for a droplet exchanging mass, as shown by the above derivation, because Newton's law, as expressed by Eq. (19), is valid only for a fixed quantity of mass (a system).
ENERGY EQUATION

The first law of thermodynamics for a system states that

$$
\dot{Q}-\dot{W}=\frac{d E}{d t},
$$

where $E$ is the energy of the system, both thermal and mechanical; that is,

$$
E=I_{d}+\text { K.E. + P.E. }
$$

and $I_{d}$ is the internal energy of the droplet. Application of the transport theorem gives

$$
\begin{aligned}
\frac{d E}{d t}= & \frac{d}{d t} \int_{c \cdot v \cdot} e_{d} d V \\
& +\int_{c . s .} \rho_{v} e w_{\alpha}^{n} \alpha d A,
\end{aligned}
$$

with e being the energy unit mass and equal to

$$
e=1_{d}+\frac{u^{2}}{2}+g z \text {, }
$$

where $1_{d}$ is the internal energy per unit mass, $u$ is the velocity magnitude with respect to an inertial reference frame, and $z$ is the elevation.

The work done by the system at the boundary in moving the flow across the control surface (flow work) is

$$
\dot{\mathrm{w}}_{\mathrm{f}}=\int_{\text {c.s. }} \mathrm{p} \mathrm{w}_{\alpha} \mathrm{n}_{\alpha} \mathrm{dA},
$$


which when combined with the efflux term In the transport theorem gives

$$
\begin{aligned}
& \dot{Q}_{d}- \dot{w}_{s}=\frac{d}{d t} \int_{c \cdot v \cdot}\left(1_{d}+\frac{u^{2}}{2}+g z\right) \rho_{d} d v \\
&+\int_{c \cdot s \cdot} \rho_{v}\left(h_{s}+\frac{\left(u_{\alpha}+w_{\alpha}\right)\left(u_{\alpha}+w_{\alpha}\right)}{2}+g z\right) \\
& w_{\gamma}{ }^{n} \gamma^{d A},
\end{aligned}
$$

where $\dot{h}_{s}$ is shaft power and $h_{s}$ is the specific enthalpy of the gases at the control surface.

The droplet is moving with a velocity $u_{1}$ with respect to inertiel space; assume it is not rotating. The inclusion of rotation is straight-forward but cumbersome. Also, for clarity, the potential energy term w1ll be neglected as 1 it is generally small compared to the thermal energles.

The shaft power performed by the droplet on the vapor is given by

$$
\dot{\mathrm{w}}_{\mathrm{s}}=-\mathrm{F}_{\mathrm{d}, \alpha} \mathrm{u}_{\alpha} \text {. }
$$

If the drag force $F_{d, 1}$ is in the direction of the droplet velocity $u_{1}$, then the vapor is accelerating the droplet so the vapor is doing work on droplet; hence, the minus sign.

Because $u_{1}$ is constant throughout the droplet, one can write*

$$
\begin{aligned}
& \frac{d}{d t} \int_{c \cdot v \cdot} \rho_{d}\left(1_{d}+\frac{u^{2}}{2}\right) d v \\
& =\frac{d}{d t}\left[m 1_{d}+m \frac{u_{\alpha} u_{\alpha}}{2}\right],
\end{aligned}
$$

*The specific internal energy is averaged over the droplet. If the droplet is small, the internal energy is uniform throughout. which ylelds

$$
\begin{gathered}
\frac{d}{d t}\left[m-d t+m \frac{u_{\alpha} u_{\alpha}}{2}\right]=m \frac{d 1_{d}}{d t}+1_{d} \frac{d m}{d t} \\
+\frac{u^{2}}{2} \frac{d m}{d t}+m u_{\alpha} \frac{d u_{\alpha}}{d t} .
\end{gathered}
$$

The magnitude of the velocity at the surface of the droplet is

$$
\frac{\left(u_{\alpha}+w_{\alpha}\right)\left(u_{\alpha}+w_{\alpha}\right)}{2}=\frac{u^{2}}{2}+\frac{w^{2}}{2}+u_{\alpha} w_{\alpha} .
$$

Assuming the spectfic enthalpy and $w$ are constant over the surface of the droplet and using the continuty equation, one can write the efflux term as

$$
\begin{aligned}
& \int_{c . s .} \rho_{v}\left(h_{s}+\frac{\left(u_{\alpha}+w_{\alpha}\right)\left(u_{\alpha}+w_{\alpha}\right)}{2} w_{\beta} n_{\beta} d A\right. \\
& =\left(h_{s}+\frac{u^{2}}{2}+\frac{w^{2}}{2}\right)\left(-\frac{d m}{d t}\right) \\
& +u_{\alpha} \int_{c: s .} w_{\alpha} \rho_{v} w_{\gamma} n_{\gamma}^{d A},
\end{aligned}
$$

and the last term reduces to zero:

$$
\int_{c . s .} w_{1} \rho_{v} w_{\alpha} n_{\alpha} d A=\rho_{v} w^{2} \quad \int_{c . s .} n_{1} d A=0 .
$$

Combining all the terms in Eq. (26) results in

$\dot{Q}_{d}=m \frac{d i_{d}}{d t}-\frac{d m}{d t}\left(h_{s}-1_{\alpha}+\frac{w^{2}}{w}\right)$

The energy equation generally found in the literature for a droplet is

$$
\dot{Q}_{d}=m c_{d} \frac{d T}{d t}-h_{f_{g}} \frac{d m}{d t} .
$$


One notes that this is a reasonably good approximation to the correct equation because $w^{2} / 2$ is usually small compared to the thermal energy terms and $i_{d}$ is very nearly the enthalpy of the Iiquid phase. Situations may arise however in which the approximate equation, Eq. (33), is not valid.

\section{Eulerian Equations for a Droplet Cloud}

As stated above, the mechanical and thermal properties of a moving droplet are most conventently described using the Lagrangian approach; that is, following Individual droplets with time. However, when one is concerned with a mixture of droplets and vapor, it becomes more convenient to regard the droplets as a continuum and define average properties, such as, the mass of droplets per unit volume of mixture. Thus the volume over which the averaging is performed must be large enough to have a stationary average according to the work of Drew. ${ }^{8}$ For purposes of analysis here, we will assume that volumes over which we write the integral equations are sufficiently large to define average droplet conditions.

Void fraction is defined as the fraction of mixture volume occupied by the vapor or

$$
\theta=\frac{v_{v}}{V},
$$

where $v_{v}$ is the volume of the vapor and $v$ is the volume of the mixture. Therefore, the volume of droplets per unit volume of mixture is

$$
\frac{v_{d}}{v}=1-\theta,
$$

and the bulk density, $\rho_{d}$, is given by

$$
\rho_{d}^{\prime}=\rho_{d}(1-\theta)
$$

where $\rho_{d}$ is the material density of the droplet.

The transport theorem has to be viewed In a somewhat different sense to treat the motion of a group of droplets through a control volume. When treating a group of droplets, one is unable to define a continuous surface which defines the interface between the system and the surroundings. Thus the transport theorem must be applied to a given identifiable group of droplets (a given number of droplets) whose mass may or may not vary with time.

Consider $\mathrm{N}$ droplets moving along a channel, as shown in Fig. 3. At time $t$, $\Delta \mathrm{N}_{\text {in }}$ droplets have yet to cross the control surface into the control volume and at time $t+\delta_{t}, \Delta \mathrm{N}_{\text {out }}$ droplets have left the control volume. Assume that each droplet has associated with it a property $b$, such as mass, so the net change in property $b$ of all the droplets in time $\delta t$ is

$$
\begin{aligned}
& \Delta(\mathrm{Nb})_{\delta t}=\left(N_{c \cdot v \cdot}\right)_{t+\delta t}-\left(N_{c \cdot v \cdot}\right)_{t} \\
& +\left(\Delta \mathrm{N}_{\text {out }} \mathrm{b}_{\text {out }}\right)-\left(\Delta \mathrm{N}_{\text {in }} \mathrm{b}_{\text {in }}\right),
\end{aligned}
$$


(a)

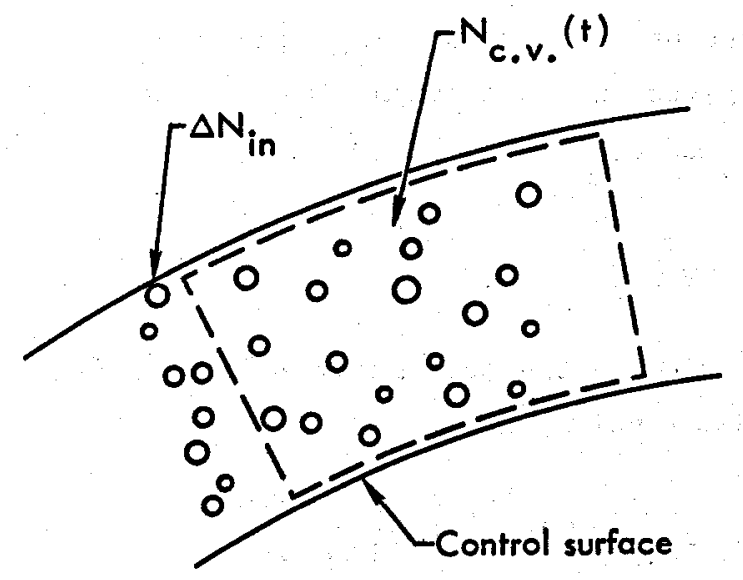

(b)

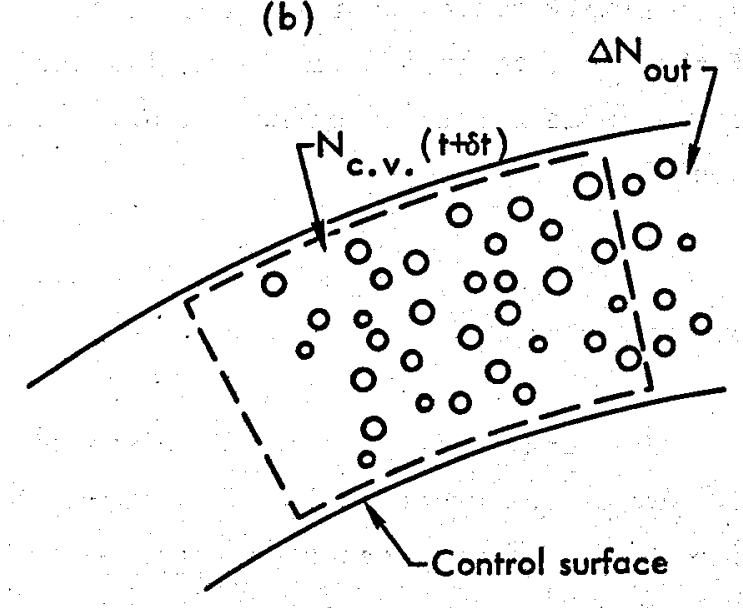

Fig. 3. Droplet motion with respect to control volume: (a) droplets at time $t$, (b) droplets at time $t+\delta t$.

where $\mathrm{N}_{\mathrm{c} . \mathrm{v} \text {. }}$ is the number of droplets in the control volume. Taking the limt as $\delta t+0$, the rate of change of property $\mathrm{Nb}$ with time is

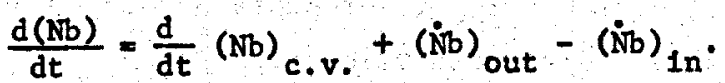

This equation is valid even if the droplets disappear (through complete evaporation, for example) because they are still assumed to "exist" but have zero value for property $b$.

\section{CONTINUITY EQUATION}

To derive the continuity equation, we simply let b be the mass of a droplet. Also, by definition,

$$
(\mathrm{Nm})_{c . v .}=\int_{c \cdot s_{.}} \rho_{d}^{\prime} d v=\int_{c . v .} \rho_{d}(1-\theta) d v .
$$

The $\dot{\mathrm{N}} \mathrm{m}$ terms in the transport equations represent the mass flux of all droplets across the control surface. Defining $U_{1}$ as the mass-averaged velocity of the droplets with respect to the control surface, one has

$$
(\dot{N} m)_{\text {out }}-\left(\dot{N}_{m}\right)_{\text {In }}=\int_{c . s .} \rho_{\mathrm{d}}^{\prime} u_{\alpha} \mathrm{n}_{\alpha}^{\mathrm{dA}},
$$

where $n_{1}$ is the unit outward normal vector. The $d(\mathrm{Nm}) / d t$ term of the transport equation represents the rate of change of mass of the droplets due to condensation or evaporation and will be regarded as mass source (or sink) terms. Thus,

$$
\frac{d}{d t}(N m)=-s_{m}=-s_{m} v,
$$

where $s_{m}$ is the rate of mass addition per untt volume of mixture due to evaporation. If evaporation is occurring, $s_{m}$ is positive; if condensation is occurring, it is negative.

Finally, the continufty equation is

$$
-s_{m}=\frac{d}{d t} \int_{c \cdot v_{0}} \rho_{d}^{\prime} d v+\int_{c . s .} \rho_{d}^{\prime} u_{\alpha} \alpha^{d A}
$$

which states that the net efflux of droplet mass from the control volume plus the rate of change of mass within the control volume 1s equal to the rate of mass change of the 
droplets due to condensation or evapora-

tion. The corresponding differential equation, assuming once again average properties are definable, is

$$
\begin{aligned}
& \frac{\partial}{\partial t}\left[\rho_{d}(1-\theta)\right] \\
& \quad+\frac{\partial}{\partial x_{\alpha}}\left[\rho_{d}(1-\theta) u_{\alpha}\right]=-s_{m} .
\end{aligned}
$$

MOMENTUM EQUATION

To derive the momentum equation, one takes $b$ equal to the momentum of a droplet; that is,

$$
b=m u_{1}
$$

where $u_{i}$ is the droplet velocity with respect to an inertial reference frame. If the droplets have different velocities, then $u_{1}$ is the local momentum-averaged velocity; that is, the momentum of a local group of droplets is equal to their cumulative mass times $u_{1}$. Thus the momentum equation becomes

$$
\begin{aligned}
\frac{d}{d t}\left(N_{i}\right)= & \frac{d}{d t} \int_{c \cdot v \cdot} \rho_{d}^{\prime} u_{i} d v \\
& +\int_{c \cdot s .} \rho_{d}^{\prime} u_{i} u_{\alpha} n_{\alpha} d A
\end{aligned}
$$

where the term on the left hand side of the equation is the rate of momentum change of the droplets (Lagrangian approach) and the first term on the right hand side is the rate of momentum change in the control volume (Eulerian approach). The corresponding differential equation is

$$
\begin{aligned}
{ }^{-s_{m}} u_{1} & +\frac{N m}{y} \frac{d u_{1}}{d t} \\
& =\frac{\partial}{\partial t}\left(\rho_{d}^{\prime} u_{1}\right)+\frac{\partial}{\partial x_{\alpha}}\left(\rho_{d}^{\prime} u_{1} u_{\alpha}\right)
\end{aligned}
$$

The second term on the left is simply the force per unit volume acting on the droplets due to aerodynamic drag and is defined as

$$
\frac{N m}{V} \frac{d u_{1}}{d t}=f_{d, 1} .
$$

Subtracting the continuity equation, Eq. (43), results in

$$
f_{d, i}=\rho_{d}^{\prime} \frac{\partial u_{i}}{\partial t}+\rho_{d}^{\prime} u_{\alpha} \frac{\partial u_{i}}{\partial x_{\alpha}},
$$

which simply states that the acceleration of the droplets in a unit volume is equal to the force per unit volume acting on the droplets.

\section{ENERGY EQUATION}

The total energy of the droplets is the sum of the mechanical and thermal energies. For purposes of clarity, only the kinetic energy contribution to the mechanical energy is included here. Should other forms be significant, such as potential or surface tension energies, they can be easily incorporated. Thus, the energy of the group of droplets is

$$
E=N m\left(i_{d}+\frac{u^{2}}{2}\right) \text {, }
$$

where $u$ is the speed of the droplets with respect to an inertial reference frame. More specifically, if droplets of different mass have different velocities, then $u^{2} / 2$ must be welghted to yield the local kinetic energy when multiplied by the local cumulative (average) mass. The same definition also applies to the internal energy ${ }^{1} d$. Substitution of droplet energy into the transport equation yields 


$$
\begin{gathered}
\frac{d}{d t}\left[N m\left(1_{d}+\frac{u^{2}}{2}\right)\right]=\frac{d}{d t} \int_{c \cdot v \cdot} \rho_{d}^{\prime} \\
\left(1_{d}+\frac{u^{2}}{2}\right) d v+\int_{c . s .} \rho_{d}^{\prime} \\
\left(1_{d}+\frac{u^{2}}{2}\right) u_{\alpha}{ }^{n} d A
\end{gathered}
$$

The corresponding differential equation, assuming as always that the averages are definable and the derivatives are continuous, is

$$
\begin{aligned}
-\left(1_{d}\right. & \left.+\frac{u^{2}}{2}\right) s_{m}+\frac{N m}{v} \frac{d}{d t}\left(1_{d}+\frac{u^{2}}{2}\right) \\
= & \frac{\partial}{\partial t}\left[\rho_{d}^{\prime}\left(1_{d}+\frac{u^{2}}{2}\right)\right] \\
& +\frac{\partial}{\partial x_{\alpha}}\left[\rho_{d}^{\prime}\left(1_{d}+\frac{u^{2}}{2}\right) u_{\alpha}\right] .
\end{aligned}
$$

Subtracting out the continulty equation leaves

$$
\begin{aligned}
\frac{N m}{V} \frac{d}{d t} & \left(1_{d}+\frac{u^{2}}{2}\right)=\rho_{d}^{\prime} \frac{\partial}{\partial t}\left(1_{d}+\frac{u^{2}}{2}\right) \\
& +\rho_{d}^{\prime} v_{\alpha} \frac{\partial}{\partial x_{\alpha}}\left(1_{d}+\frac{u^{2}}{2}\right)
\end{aligned}
$$

Also, recognizing that

$$
u^{2}=u_{\alpha} u_{\alpha}
$$

so that

$$
\frac{d u^{2}}{d t}=2 u_{\alpha} \frac{d u_{\alpha}}{d t}
$$

and

$$
\rho_{d}^{\prime} v_{\alpha} \frac{\partial}{\partial x_{\alpha}}\left(\frac{u^{2}}{2}\right)=\rho_{d}^{\prime} v_{\alpha} u_{\beta} \frac{\partial u_{\beta}}{\partial x_{\alpha}}
$$

the momentum equation, Eq. (46), can be subtracted to leave

$$
\frac{N m}{v} \frac{d 1 d}{d t}=\rho_{d}^{\prime} \frac{\partial 1_{d}}{\partial t}+\rho_{d}^{\prime} v_{\alpha} \frac{\partial 1_{d}}{\partial x_{\alpha}} .
$$

It is important to note here that the enthalpy of the droplet does not appear because the droplets do not contribute to flow work; that 1s, one droplet is incapable of doing flow work on another. Several derivations falsely include a flow work for the droplets - a fallacy arising from regarding the discrete droplets as a continuum.

Using the results of the previous section for the energy equation of a single droplet, one can write

$$
\begin{aligned}
\dot{q}_{d} & -s_{\text {II }}\left(h_{s}+\frac{w^{2}}{2}-1_{d}\right) \\
& =\rho_{d}^{\prime} \frac{\partial 1_{d}}{\partial t}+\rho_{d}^{\prime} v_{\alpha} \frac{\partial 1_{d}}{\partial x_{\alpha}},
\end{aligned}
$$

where $\dot{q}_{d}$ is the heat transfer rate to the droplets per unit volume. Thus we see that the rate of energy change of the droplets in a unit volume is equal to the rate of heat transfer to the droplets minus the energy convected away due to evaporation.

It is Important to note that no conservation principles, such as conservation of mass, Newton's second law, and the first law of thermodynamics, were used in the derivation of the above equations. The Lagrangian description of droplet property variations is simply converted to an Eulerian description. 


\section{Eulerian Equations for Vapor Phase}

Consider a group of droplets enclosed by an arbitrary surface $S$ which may, or may not, intersect some droplets, as shown in Fig. 4. That portion of $S$ which intersects the "boundary" droplets is Identifled as $\delta S$. The surface surrounding and adjacent to every interior droplet is $\mathrm{Sp}$ and the portion of surface surrounding the "boundary" droplets which is interior to $S$ is defined a $\delta S_{p}$. Thus the control surface for the vapor phase is $S+s_{p}-\delta s-\delta s_{p}$.

Vold fraction can also be used to relate the density of vapor per unit volume of mixture to the actual vapor density,

$$
\rho_{v}^{\prime}=\rho_{v}^{\theta}
$$

where $\rho_{v}^{\prime}$ is the vapor density of the mixture and $\rho_{v}$ is the actual vapor density, a thermodynamic property.

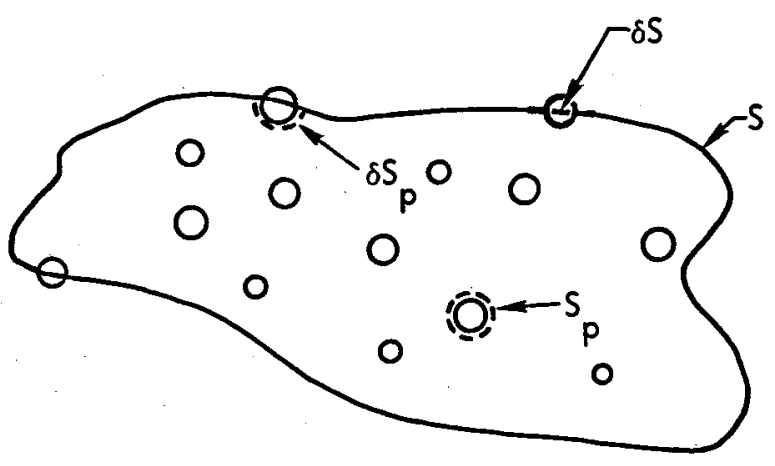

Fig. 4. Control volume for vapor phase.
CONTINUITY EQUATION

The conservation of mass as given by the transport theorem is

$\frac{d}{d t} \int_{c . v .} \rho_{v}^{\prime} d V+\int_{c . s .} \rho_{v} v_{\alpha}{ }^{n} d A=0$,

where $v_{i}$ is the velocity relative to the control surface. For the control surface of the vapor as depicted in Fig. 4, we have

$$
\begin{aligned}
& \frac{d}{d t} \int_{c, v .} \rho_{v}^{\prime} d v+\int_{S-\delta S} \rho_{v} v_{\alpha}{ }^{n} \alpha d A \\
& +\int_{s_{p}+\delta S_{p}} \rho_{v} w_{\alpha}{ }^{n} d A=0 \text {, }
\end{aligned}
$$

where $n_{1}$ is the unit outward normal vector which, on the surface $s_{p}+\delta s_{p}$, is directed toward the interior of the droplets. Also V is the volume enclosed by the surface $S$. By definition of mixture density of the vapor, one can write

$$
\int_{S-\delta S} \rho_{v} v_{\alpha^{n} \alpha} d A=\int_{S} \rho_{v}^{\prime} v_{\alpha}{ }^{n} d A
$$

Also from the continuity equation for the droplets, one realizes that

$$
\int_{p}+\delta s_{p} \rho_{v} \cdot w_{\alpha} n_{\alpha} d A=-s_{m}
$$

where $S_{m}$ is the rate of mass flow from the droplets. If the droplets are evaporating, $S_{m}$ is a positive number. 
The final form of the integral

equation is

$$
\frac{d}{d t} \int_{V} \rho_{v}^{\prime} d V+\int_{S} \rho_{v}^{\prime} v_{\alpha}{ }^{n} d A=s_{m},
$$

which simply expresses the fact that the rate of increase of vapor mass inside $V$ plus the net efflux of vapor from $S$ is equal to the rate of vapor mass addition by the droplets. The corresponding differential equation is

$$
\frac{\partial}{\partial t} \rho_{v}^{\prime}+\frac{\partial}{\partial x_{\alpha}}\left(\rho_{v}^{\prime} v_{\alpha}\right)=s_{m}
$$

where, as before, it is assumed that averages can be defined and the derivatives are continuous.

\section{MOMENTUM EQUATION}

Let us define $u_{1}$ as the local velocity of the droplets (momentum-averaged) w1th respect to an Inertial reference frame. Thus the velocity of the vapor at the droplet surface with respect to an Inertial reference frame is $u_{1}+w_{1}$.

It is also necessary to consider in more detall the mass flux from and the forces on the "boundary" droplets. At any given time, droplets along a boundary will be in various stages of penetration through the boundary, as shown in Fig. 5 . In the figure, droplet $A$ is almost completely inside the surface $S$ while droplet $B$ has just made contact with $S$. It is reasonable to postulate that "on the average" the combination of droplets $B$ and $C$ can be treated as one droplet inside $S$ and the other outside of $S$. Thus, by averaging, one should be able to stipulate
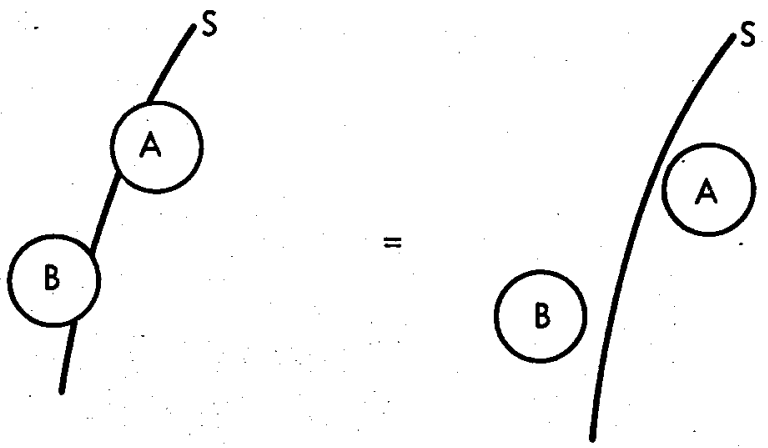

Fig. 5. Droplets in various stages of penetrating through the boundary (for any given time).

that droplets more than half way across $S$ are considered inside and those less than half way across are regarded as outside.

Using the transport equation for the momentum of the fluid phase, we have

$$
\begin{aligned}
F_{1}= & \frac{d}{d t} \int_{v} \rho_{v}^{\prime} v_{1} d v+\int_{s-\delta S} \rho_{v} v_{1} v_{\alpha} n_{\alpha} d A \\
& +\int_{s}+\delta S_{p} \rho_{v}\left(w_{1}+u_{1}\right) w_{\alpha} n_{\alpha} d A
\end{aligned}
$$

where it is assumed that $S$ is an inertial reference frame and $v_{1}$ is measured with respect to $S$. In view of the above argument, the surface $s_{p}+\delta s_{p}$ can be modified to include all droplets as being defined "Inside" the control volume. Thus we w111 regard $s_{p}+\delta s_{p}$ as $s_{p}^{\prime}$ indicating Inclusion of the "Inside" boundary droplets. Thus we can write the momentum equation as

$$
\begin{aligned}
F_{1}= & \frac{d}{d t} \int_{V} \rho_{v} v_{1} d V+\int_{S} \rho_{v}^{\prime} v_{1} v_{\alpha} d \alpha d A \\
& +\int_{S_{p}^{\prime}} \rho_{v}\left(w_{1}+u_{1}\right) w_{\alpha} n_{\alpha} d A
\end{aligned}
$$


Because $u_{i}$ is constant over the surface of the droplet, one can write

$$
\int_{s_{p}^{\prime}} u_{1} \rho_{v} w_{\alpha}{ }^{n} d A=-u_{1} s_{m}
$$

from the continuity equation where $S_{m}$ is the evaporation rate (or if negative condensation rate) in the volume V. Also if the mass flux is uniform over the surface of the droplet, one has

$\int_{s_{p}^{\prime}} \rho_{v} w_{i} w_{\alpha} n_{\alpha} d A=\rho_{v} w^{2} \int_{s_{p}^{\prime} n_{1}} d A=0$.

The forces acting on the vapor are the pressure and shear forces on the surface $S-\delta S$ and the aerodynamic drag forces on the surface $S_{p}^{\prime}$. For clarity, other forces, such as gravitationsl, are not included. Finally, one can write the integral form of the momentum equation as

$$
\begin{aligned}
-F_{d, 1} & +\int_{S} \theta T_{1 \alpha} n_{\alpha} d A-\int_{S} \theta p n_{i} d A \\
= & \frac{d}{d t} \int_{V} \rho_{v}^{\prime} v_{1} d V+\int_{S} \rho_{v}^{\prime} v_{i} v_{\alpha} n_{\alpha} d A \\
& -u_{i} s_{m},
\end{aligned}
$$

where $F_{D, 1}$ is the cumulative aerodynamic drag force on the droplets in volume $V$, $\tau_{1 f}$ is the shear stress in the 1 -direction due to the surface represented by normal $n_{j}$, and $p$ is the pressure. The void fraction in the shear-stress and pressureforce integrals represents that fraction of $S$ which is in the vapor phase. The corresponding differential equation is

$$
\begin{aligned}
\frac{\partial}{\partial t}\left(\rho_{v}^{\prime} v_{1}\right)+\frac{\partial}{\partial x_{\alpha}}\left(\rho_{v}^{\prime} v_{1} v_{\alpha}\right)+\frac{\partial}{\partial x_{1}}\left(\theta_{p}\right) \\
=-f_{D, 1}+\frac{\partial}{\partial x_{\alpha}}\left(\theta \tau_{1 \alpha}\right)+s_{m} u_{i},
\end{aligned}
$$

where $f_{D, 1}$ and $s_{m}$ are the drag force and vapor mass addition per unit volume of mixture. Subtracting the continuity equation derived above; the momentum equation can be rewritten as

$$
\begin{aligned}
\rho_{v}^{\prime} \frac{\partial v_{1}}{\partial t} & +\rho_{v}^{\prime} v_{\alpha} \frac{\partial v_{1}}{\partial x_{\alpha}}+\frac{\partial}{\partial x_{1}}\left(\theta_{p}\right) \\
= & -f_{D, 1}+\frac{\partial}{\partial x_{\alpha}}\left(\theta \tau_{1 \alpha}\right) \\
& +s_{m}\left(u_{1}-v_{1}\right)
\end{aligned}
$$

and we note that with the momentum equation In this form the momentum source term due to mass addition depends on the dropletvapor velocity difference.

\section{ENERGY EOUUATIONS}

The energy of the vapor phase consists of thermal and mechanical energies. As before, for sake of clartty, we will only consider the internal and kinetic energies. Generally the gravitational potential energy is small compared to the thermal energy. If the droplets are very small (less than a $\mu \mathrm{m}$ ), surface tension energy may also have to be included.

The energy per unit mass is

$$
e=1_{v}+\frac{v^{2}}{2}
$$

when $v$ is the vapor speed with respect to an Inertial reference frame. Applying the 
first law of thermodynamics to the system, we have

$$
\dot{Q}-\dot{\mathrm{W}}=\frac{\mathrm{d}}{\mathrm{dt}}(\mathrm{eM})
$$

Utilizing the transport theorem, we have

$$
\begin{aligned}
\dot{Q}-\dot{W}= & \frac{d}{d t} \int_{v} \rho_{v}^{\prime} e d v+\int_{s-\delta s} \rho_{v} e v_{\alpha}^{n} d^{d A} \\
& +\int_{S_{p}^{\prime}} \rho_{v} e w_{\alpha}^{n} \alpha d A .
\end{aligned}
$$

The work term involves work which is done against pressure and shear stress on the boundary.

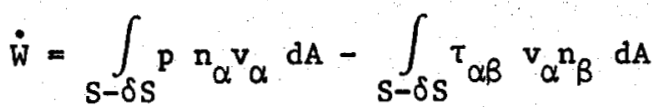

$$
\begin{aligned}
& +\int_{S_{p}^{\prime}} p n_{\alpha}\left(w_{\alpha}+u_{\alpha}\right) d A-\int_{s_{p}^{1}} \tau_{\alpha \beta}\left(w_{\alpha}+u_{\alpha}\right) n_{\beta} d A
\end{aligned}
$$

The first pressure integral can be combined with the efflux term to yield the enthalpy of the gas and represents the flow work in the usual fashion.

$$
\begin{aligned}
& \int_{s-\delta S}\left(p v_{\alpha}+\rho_{v} v_{\alpha} e\right) n_{\alpha} d A \\
& =\int_{S} \rho_{v}^{\prime}\left(h_{v}+\frac{v^{2}}{2}\right) v_{\alpha} n_{\alpha} d A
\end{aligned}
$$

The integrals over the droplet surface can be rewritten as

$$
\begin{aligned}
\int_{s_{p}^{\prime}} p n_{\alpha}\left(w_{\alpha}+u_{\alpha}\right) d A-\int_{s_{p}^{\prime}} \tau_{\alpha \beta}\left(w_{\alpha}+u_{\alpha}\right) n_{\beta} d A \\
=u_{\alpha} \int_{s_{p}^{\prime}}\left(p_{\alpha}-\tau_{\alpha \beta} n_{\beta}\right) d A \\
+\int_{s_{p}^{\prime}} p n_{\alpha} w_{\alpha} d A-\int_{s_{p}^{\prime}} \tau_{\alpha \beta} w_{\alpha \beta} n^{n_{B}} d A
\end{aligned}
$$

One recognizes the first integral on the left as the shear and pressure forces on the droplets (remember $n_{1}$ is inward to the droplet) or, simply, the aerodynamic force on the droplet, so

$$
u_{\alpha} \int_{s_{p}^{\prime}}\left(p_{\alpha}-\tau \tau_{\alpha \beta} n_{\beta}\right) d A=u_{\alpha} F_{d, \alpha} .
$$

The second integral on the left in Eq. (71) can be combined with efflux of energy term from the droplet surface in $\mathrm{Eq}$. (68) to convert internal energy to enthalpy at the droplet surface. The third integral is zero because $\tau_{1 j}$ is normal to $w_{1} *$. The energy efflux term at the droplet surface incorporating the flow work due to mass flow from the vapor-droplet interface is

$$
\begin{aligned}
& \int_{s_{p}^{\prime}} \rho_{v}\left(e+\frac{p}{\rho_{v}}\right) w_{\alpha} n_{\alpha} d A \\
& =\int_{S_{p}^{1} \rho_{v}}\left(h_{s}+\frac{u^{2}}{2}+\frac{w^{2}}{2}+w_{\alpha} u_{\alpha}\right) w_{B} n_{B} d A .
\end{aligned}
$$

Assuming as before that $h_{s}$ and $w_{1}$ are constant over the droplet surface, the droplet efflux term becomes

*Th1s can be proved by setting $w_{1}=w_{1}$ so

$$
\begin{aligned}
& \tau_{\alpha \beta} w_{\alpha \beta} n_{\beta}=w \tau_{\alpha \beta} n_{\alpha \beta} n=w \tau_{\alpha \beta} \delta_{\alpha \beta} \\
& =w \tau_{\alpha \alpha}=0 .
\end{aligned}
$$

where $\delta_{i f}$ is the Kronecker delta $\left(\delta_{i j}=0\right.$ if $1 \neq j$ and $\delta_{1 j}=1$ if $i=j$ ) and $\tau_{\alpha \alpha}$ is the sum of the shear stress components normal to surface, which by definition is zero. 


$$
\begin{aligned}
\int_{s_{p}^{\prime}} \rho_{v} & \left(e+\frac{p}{\rho_{v}}\right) w_{\alpha} n_{\alpha} d A \\
& =-s_{m}\left(h_{s}+\frac{u^{2}}{2}+\frac{w^{2}}{2}\right),
\end{aligned}
$$

where $S_{m}$ is the evaporation rate of the droplet.

The heat transfer term can also be broken down to two contributions

$\dot{Q}=-\int_{S-\delta S} q_{\alpha} n_{\alpha} d A-\int_{S_{p}^{\prime}} q_{\alpha} n_{\alpha} d A$,

where the first term is the heat transfer to the volume across the surface $S-\delta S$ and the second term is the heat transfer from the droplets to the gas. The heat transfer term can be rewritten as

$$
\dot{Q}=-\int_{S} \theta q_{\alpha^{n}{ }_{\alpha}} d A-v \dot{q}_{d}
$$

where, as before, the factor $\theta$ represents the fraction of $S$ through the vapor phase. Finally, the energy equation can be written in integral form as

$$
\begin{aligned}
\frac{d}{d t} \int_{S} \rho_{v}^{\prime}\left(1_{v}+\frac{v^{2}}{2}\right) d v+\int_{S} \rho_{v}^{\prime}\left(h_{v}+\frac{v^{2}}{2}\right) v_{\alpha} n_{\alpha} d A \\
=-\int_{S} \theta q_{\alpha} n_{\alpha} d A-v \dot{q}_{d} \\
+\int_{S} \theta \tau_{\alpha \beta} v_{\alpha}^{n_{B} d A} \\
+S_{m}\left(h_{s}+\frac{u^{2}}{2}+\frac{w^{2}}{2}\right)-u_{\alpha} F_{D, \alpha}
\end{aligned}
$$

and the corresponding differential

equation is

$$
\begin{gathered}
\frac{\partial}{\partial t}\left[\rho_{v}^{\prime}\left(1 v+\frac{v^{2}}{2}\right)\right]+\frac{\partial}{\partial x_{\alpha}}\left[\rho_{v}^{\prime}\left(h_{v}+\frac{v^{2}}{2}\right) v_{\alpha}\right] \\
=-\frac{\partial}{\partial x_{\alpha}}\left(\theta q_{\alpha}\right)-\dot{q}_{\alpha}+\frac{\partial}{\partial x_{B}}\left(\theta \tau_{\alpha \beta} v_{\alpha}\right) \\
+s_{m}\left(h_{s}+\frac{u^{2}}{2}+\frac{w^{2}}{2}\right)-u_{\alpha} f D, \alpha
\end{gathered}
$$

from which we see that the droplets contribute energy to the vapor by heat transfer, by mass transfer, and by work against aerodynamic drag forces. Using the momentum and energy equations for the Individual droplets, Eqs. (18) and (32), these source terms can be rewritten as

$$
\begin{aligned}
-\dot{q}_{d} & +s_{m}\left(h_{s}+\frac{u^{2}}{2}+\frac{w^{2}}{2}\right)-u_{\alpha} f_{D, \alpha} \\
& =-\frac{d}{d t}\left(\frac{N m d}{v}\right)+s_{m} \frac{u^{2}}{2}-u_{\alpha} f_{D, \alpha} \\
& =-\frac{d}{d t}\left[\frac{N m}{v}\left(i_{d}+\frac{u^{2}}{2}\right)\right]
\end{aligned}
$$

and incorporated into Eq. (78) to yield

$$
\begin{aligned}
\frac{\partial}{\partial t}\left[\rho_{v}\left(i_{v}+\frac{v^{2}}{2}\right)\right]+\frac{\partial}{\partial x_{\dot{\alpha}}}\left[\rho_{v}^{\prime}\left(h_{v}+\frac{v^{2}}{2}\right) v_{\alpha}\right] \\
=-\frac{\partial}{\partial x_{\alpha}}\left(\theta q_{\alpha}\right)+\frac{\partial}{\partial x_{\beta}}\left(\theta \tau_{\alpha \beta} v_{\alpha}\right) \\
-\frac{d}{d t}\left[\frac{N m}{v}-\left(1_{d}+\frac{u^{2}}{2}\right)\right],
\end{aligned}
$$

which fllustrates how a loss of energy in the liquid phase represents a source of energy to the vapor phase. 


\section{Mixture Equations}

The addition of the droplet and vapor-phase equations should yield. the mixture equations. The mfxture equations are easily derived from first principles, so the equations resulting from adding the 1iquid and vapor phase equations provide a check as to the validity of each.

\section{CONTINUITY EQUATION}

The continuity equations for the liquid and vapor phases are Eq. (43),

$$
\frac{\partial \rho_{d}^{\prime}}{\partial t}+\frac{\partial}{\partial x_{\alpha}}\left(\rho_{d}^{\prime} u_{\alpha}\right)=-s_{m},
$$

and $\mathrm{Eq} \cdot(58)$,

$$
\frac{\partial \rho_{v}^{\prime}}{\partial t^{\prime}}+\frac{\partial}{\partial x_{\alpha}}\left(\rho_{v}^{\prime} v_{\alpha}\right)=s_{m}
$$

Adding these two equations, one finds the source terms cancel to give

$\frac{\partial}{\partial t}\left(\rho_{d}^{\prime}+\rho_{v}^{\prime}\right)+\frac{\partial}{\partial x_{\alpha}}\left(\rho_{d}^{\prime} u_{\alpha}+\rho_{v}^{\prime} v_{\alpha}\right)=0$,

which states that the mass of the mixture is conserved.

\section{MOMENTUM EQUATION}

The momentum equations for the Ilquid and vapor phases are Eq. (45),

$$
\begin{gathered}
\frac{\partial}{\partial t}\left(\rho_{d}^{\prime} u_{1}\right)+\frac{\partial}{\partial x_{\alpha}}\left(\rho_{d}^{\prime} u_{1} u_{\alpha}\right) \\
=-s_{m} u_{1}+f_{D, 1}
\end{gathered}
$$

and $\mathrm{Eq} .(64)$,

$$
\begin{aligned}
\frac{\partial}{\partial t}\left(\rho_{v}^{\prime} v_{1}\right) & +\frac{\partial}{\partial x_{\alpha}}\left(\rho_{v}^{\prime} v_{1} v_{\alpha}\right) \\
= & -\frac{\partial}{\partial x_{1}}(\theta p)+\frac{\partial}{\partial x_{\alpha}}\left(\theta \tau_{1 \alpha}\right) \\
& +s_{m} u_{1}-f_{D, 1} .
\end{aligned}
$$

Adding these two equations, one obtains

$$
\begin{gathered}
\frac{\partial}{\partial t}\left(\rho_{d}^{\prime} u_{1}+\rho_{v}^{\prime} v_{i}\right)+\frac{\partial}{\partial x_{\alpha}}\left(\rho_{d}^{\prime} u_{i} u_{\alpha}+\rho_{v}^{\prime} v_{i} v_{\alpha}\right) \\
=-\frac{\partial}{\partial x_{1}}(\theta p)+\frac{\partial}{\partial x_{\alpha}}\left(\theta \tau_{1 \alpha}\right),
\end{gathered}
$$

which is the momentum equation for the mixture. One feature of this equation which may not be readily apparent in direct derivation of the mixture equations is the $\theta$ factor in the pressure and shear stress terms which result from the effective area of the vapor being reduced by the

presence of the droplets.

ENERGY EQUATIONS

The energy equations for the liquid and vapor phases are Eq. (48),

$$
\begin{gathered}
\left.\frac{\partial}{\partial t}\left[\rho_{d}^{\prime}\left(1_{d}+\frac{u^{2}}{2}\right)\right]+\frac{\partial}{\partial x_{\alpha}}\left[\rho_{d}^{\prime} 1_{d}+\frac{u^{2}}{2}\right) u_{\alpha}\right] \\
=\frac{d}{d t}\left[\frac{N m}{v}\left(1_{d}+\frac{u^{2}}{2}\right)\right]
\end{gathered}
$$


and Eq. (80),

$$
\begin{gathered}
\left.\left.\frac{\partial}{\partial t}\left[\rho_{v}^{\prime}\left(i_{v}+\frac{v^{2}}{2}\right)\right]+\frac{\partial}{\partial x_{\alpha}}\right] \rho_{v}^{\prime}\left(h_{v}+\frac{v^{2}}{2}\right) v_{\alpha}\right] \\
=-\frac{d}{d t}\left[\frac{N m}{v}\left(1_{d}+\frac{u^{2}}{2}\right)\right]-\frac{\partial}{\partial x_{\alpha}}\left(\theta q_{\alpha}\right) \\
+\frac{\partial}{\partial x_{\beta}}\left(\theta \tau_{\alpha \beta} v_{\alpha}\right) .
\end{gathered}
$$

Adding these two equations to eliminate the source terms ylelds

$$
\begin{aligned}
\frac{\partial}{\partial t} & {\left[\rho_{d}^{\prime}\left(1_{d}+\frac{u^{2}}{2}\right)+\rho_{v}^{\prime}\left(1_{v}+\frac{v^{2}}{2}\right)\right] } \\
& +\frac{\partial}{\partial x_{\alpha}}\left[\rho_{d}^{\prime} v_{\alpha}\left(1_{d}+\frac{u^{2}}{2}\right)\right. \\
& \left.+\rho_{v}^{\prime} v_{\alpha}\left(h_{v}+\frac{v^{2}}{2}\right)\right] \\
= & -\frac{\partial}{\partial x_{\alpha}}\left(\theta q_{\alpha}\right)+\frac{\partial}{\partial x_{\beta}}\left(\theta \tau_{\alpha \beta} v_{\alpha}\right) .
\end{aligned}
$$

Thus; one finds that the energy of the mixture is altered by the heat transfer from the boundary and work rate on the boundary. Once again the factor $\theta$ appears because of the reduction in vapor area. Also one notes the internal energy, not the enthalpy, in the IIquid specific energy term because the droplets are incapable of performing flow work.

In the derivation of the vapor-phase equations, the velocity $v_{1}$ was referenced to the control surface which was assumed to be an Inertial reference frame. The velocity $u_{1}$ is the mass-average velocity of the droplets with respect to the control surface. Assuming the droplets are uniform in size allows one to substitute $u_{i}$ for $U_{1}$ since the mass-averaged, momentumaveraged, and energy-averaged velocities would be identical.

\section{Interphase Property Transfer}

The nature of a vapor-droplet flow is primarily dependent on the transfer of mass, momentum, and energy between phases. For example, droplets moving faster than the vapor transfer momentum to the vapor through aerodynamic drag. Simultaneously, by the same mechanism, the vapor slows down the droplets, the tendancy being always toward equilibrium. Similarly, heat and mass transfer alter the thermal and mechanical properties of both phases. It is this two-way coupling phenomenon that makes the analysis of two-phase flows Inordinately difficult. In developing a computational model for vapor-droplet flows, it is very important to use valid equations for interphase property transfer.

The following section discusses some of the basic features of mass, momentum, and energy transfer between phases and provides empirical equations to quantify each.

MASS TRANSFER

The primary mechanism responsible for mass transfer between phases is diffusion. The mass flux rate of specte $A$ is proportional to the density gradient through Fick's law: 


$$
(\rho v)_{A, I}=-D \frac{\partial \rho_{A}}{\partial x_{1}} \text {, }
$$

where $D$ is the diffusion coefficient $\left(\mathrm{ft}^{2} / \mathrm{sec}\right)$ and $\rho_{A}$ is the density of specie A. If one defines $\rho$ as the mixture density, then

$$
(\rho v)_{A, 1}=-\rho D \frac{\partial c_{A}}{\partial x_{1}} \text {, }
$$

when $c_{A}$ is the concentration.

The nondimensional number relating to mass transfer is the Sherwood number (sometimes referred to as the diffusion Nusselt number) and is defined as

$$
\mathrm{Sh}=\frac{\dot{\mathrm{mL}}}{\rho \mathrm{AD}\left(c_{\mathbf{s}}-c_{\infty}\right)} \text {, }
$$

where $\dot{i n}$ is the rate of mass transfer (evaporation is defined as positive), A is the surface area, and $L$ is a characteristic length. The symbols $c_{s}$ and $c_{\infty}$ refer to the concentration at the surface and at infinity (or far from the surface). The rate of evaporation of a spherfcal droplet is given by

$$
\dot{m}=\operatorname{Sh} \rho D 4 \pi d\left(c_{s}-c_{\infty}\right) \text {. }
$$

The Sherwood number depends on the Reynolds number and Schmidt number. A high relative Reynolds number will Increase the evaporation rate because the boundary layer is thinner and the concentration gradient is increased. The Schmidt number is defined as the ratio of momentum diffusivity, or kinematic viscosity, to mass diffusivity

$$
\text { Sc }=\frac{\nu}{D} \text {. }
$$

If the Schmidt number is greater than unity, then the momentum diffusivity exceeds the mass diffusivity, which implies the concentration boundary layer will be thinner than the velocity boundary 1ayer; hence, an even larger concentration gradient is present than reflected by the Reynolds number alone. A reasonably good empirical equation for the Sherwood number $1 s^{1}$

$$
\mathrm{Sh}=2+0.55 \mathrm{Re}^{1 / 2} \mathrm{Sc}^{1 / 3},
$$

which is valld for Reynolds number up to $10^{4}$.

As an example calculation, consider a water droplet, $10 \mu \mathrm{m}$ in dlameter, moving at $10 \mathrm{ft} / \mathrm{sec}$ in air. The droplet and air are both at $60^{\circ} \mathrm{F}$ and relative humidity of the alr is $40 \%$. First of all, the Reynolds number for the droplet is

$$
\operatorname{Re}=(10)\left(3.2 \times 10^{-5}\right) / 1.58 \times 10^{-4}=2.02 .
$$

The Schmldt number for water vapor in air is approximately 0.6 . Thus the Sherwood number is

$\mathrm{Sh}=2+0.55(2.02)^{1 / 2}(0.6)^{1 / 3}=3.2$.

The concentration of water vapor in air at $40 \%$ relative humidity is 0.0044 , while the concentration at the droplet surface is 0.011 . Using a density of $0.075 \mathrm{lbm} / \mathrm{ft}^{3}$ and a diffusion coefficient of $2.6 \times 10^{-4}$ $\mathrm{ft}^{2} / \mathrm{sec}$, one calculates an evaporation rate of $1.66 \times 10^{-10} \mathrm{lbm} / \mathrm{sec}$. The droplet will continue to cool due to evaporation until it reaches the wet-bulb temperature corresponding to the relative humidity in the air whlch, for this example, is approximately $48^{\circ} \mathrm{F}$. 
The mass transfer processes in a single-component two-phase flow are much more complex and dependent on heat transfer. Diffusion, as such, does not contribute to mass transfer because there is no concentration gradient. A droplet in equilibrium with the vapor will be at the saturation temperature corresponding to the ambient pressure. If the ambient pressure is suddenly lowered, as in a flashing process, and the droplet temperature has insufficient time to respond, local bolling will occur at nucleation centers inside the droplet leading to droplet disintegration. If, however, the droplet is small, thermal equilibrium will be maintained and the droplet $w 111$ evaporate at a rate just sufficient to keep its temperature equal to the local saturation temperature.

Assuming the ambient temperature varies linearly, one finds that the average droplet temperature will follow within $99 \%$ of the ambient temperature $1 f^{9}$

$$
\frac{K t}{d^{2}} \simeq 6
$$

where $K$ is the thermal diffusivity of the droplet 1iquid. Thus a characteristic time for temperature change of the droplet is

$$
t_{d}=\frac{6 d^{2}}{k}
$$

If this characteristic time is small compared to the time required to complete the pressure drop, say $t_{p}$, then droplet disintegration is unlikely to occur. In other words, if

$$
\frac{6 d^{2}}{k t_{p}}<1
$$

then the droplets should not disintegrate due to interior boiling. For example, assume water droplets are subjected to a pressure drop which takes place in $10 \mathrm{msec}$. Using the above relationship, one predicts droplet disintegration should not occur for droplets less than $15 \mu \mathrm{m}$. Simllarly for a $1 \mathrm{msec}$ pressure drop, the corresponding stable droplet slze is approximately $5 \mathrm{\mu m}$.

It is instructive at this point to evaluate the magnitude of the flux velocity $w$ at the surface of a droplet and compare it to the thermal energy terms. From the droplet energy equation, Eq. (32), we have $\dot{Q}_{d}=m \frac{d i d}{d t}-\frac{d m}{d t}\left(h_{s}-1_{d}+\frac{w^{2}}{2}\right)$.

Assuming thermal equilibrium and, to begin with, that $w^{2}$ is small compared to $h_{s}-i_{d}$ and, moreover, that $h_{s}-i_{d} \sim h_{f g}$, one can write

$$
\dot{\mathrm{m}} \sim \frac{\mathrm{mc} d}{\mathrm{~h}_{\mathrm{fg}}} \frac{\mathrm{dT} \mathrm{d}}{\mathrm{dt}}
$$

or

$$
w=-\frac{1}{6} \frac{\rho_{\mathrm{d}}}{\rho_{\mathrm{v}}} \frac{\mathrm{dc} \mathrm{d}}{\mathrm{h}_{\mathrm{fg}}} \frac{\mathrm{dT} \mathrm{d}}{\mathrm{dt}} .
$$

If the pressure drops from 500 to 2 psia In $0.5 \mathrm{msec}$, then the rate of temperature decrease is

$$
\frac{\mathrm{dT}}{\mathrm{dt}} \sim-7 \times 10^{5}{ }^{\circ} \mathrm{F} / \mathrm{sec} .
$$

The corresponding flux velocity for a 5$\mu \mathrm{m}$ droplet at 2 psia is $15 \mathrm{ft} / \mathrm{sec}$, which amounts to $0.009 \mathrm{Btu} / \mathrm{Ibm}$ and is negligible compared to the thermal energy. 
If the pressure is suddenly increased, there will be no droplet disintegration but simply a condensation of vapor on the droplet surface effecting a heating of the droplet toward the new saturation temperature. Of course, the same arguments used above w111 apply here. That is, the temperature of a $15-\mu \mathrm{m}$ droplet will essentially follow the ambient temperature if the change occurs in approximately 10 msec. If the droplets are large and cannot respond so quickly, the surface layer is Immediately heated due to local condensation and the temperature within the droplet increases more slowly with time. The condensation rate will be determined by the rate at which heat is transferred to the interior of the droplet. A review of the literature suggests 1ittle information, if any, is avallable for the condensation rate in single-component flows under such conditions.

MOMENTUM TRANSFER

As noted in the previous sections, momentum exchange between phases is accomplished by transfer of mass through a velocity difference or aerodyanmic forces. Having already treated mass transfer, we will focus here on aerodynamic drag and 1ift.

The aerodynamic forces acting on a droplet can be subdivided into six distinct phenomena: aerodynamic drag, buoyancy, Basset force, virtual mass effect, Saffman 1ift force, and the Magnus effect.
Aerodynamic Drag

Generally the steady-state aerodynamic drag is the largest force acting on a droplet in the drag-direction (parallel to the relative vapor-droplet velocity vector) and is quantified by

$F_{D, 1}=\frac{1}{2} \rho_{v} \frac{\pi d^{2}}{4} C_{D}\left|v_{1}-u_{1}\right|\left(v_{1}-u_{1}\right)$,

where $C_{D}$ is the drag coefficient. Note that the force is in the direction $\left(v_{1}-u_{1}\right)$. The drag coefficient is primarily a function of the Reynolds number based on the relative velocity but may also depend on Mach number, droplet shape, and evaporation rate.

The asphericity of a droplet will depend on the Weber number which is defined es

$$
w=\rho_{v} \frac{\left|v_{1}-u_{1}\right|^{2} d}{\sigma},
$$

where $\sigma$ is the surface tension. The Weber number can be thought of as the ratio of inertial to surface tension forces, so a high Weber number implies a tendency for the dynamic forces to overcome the surface tension forces and shatter the droplet. Generally this occurs for a Weber number near ten. Thus if the Weber number is unity or less, the droplets should be nearly spherical.

As an example, consider water droplets moving with a relative velocity of $100 \mathrm{ft} / \mathrm{sec}$ In water vapor with a density 
of $0.005 \mathrm{lbm} / \mathrm{ft}^{3}(\mathrm{p}=2 \mathrm{psia})$. Using $\mathrm{a}$ surface tension of $5 \times 10^{-3} \mathrm{lbf} / \mathrm{ft}$ and setting the Weber number equal to unity, one predicts that droplets less than 100 $\mu \mathrm{m}$ will be spherical.

Droplet evaporation tends to reduce the drag coefficient as noted from studies of accelerated burning droplets. ${ }^{10}$ The effects are significant only at extremely high burning rates, such as for droplets burning in pure oxidizing atmospheres, so evaporation effects should be insignificant for geothermal applications.

Mach number (relative Mach number) effects depend on the magnitude of the Reynolds number. If the Reynolds number is greater than 50, an increasing Mach number tends to increase the drag coefficient while the opposite trend is observed for Reynolds numbers less than 50. The magnitude of the Mach number effect on drag coefficient is small provided $M<$ 0.4 unless the Reynolds number is also small, in which case rarefied flow conditions are approached. The degree of rarefaction is quantified by the Knudsen number, or the ratto of mean free path to particle diameter:

$$
\mathrm{Kn}=\frac{\lambda}{\mathrm{d}} \cdot
$$

If the Knudsen number is greater than unity, the flow is identified as a freemolecule flow and the drag coefficient for continuum flow is no longer valid.

Rarefaction effects can still be significant for Knudsen numbers one-tenth or less when the droplet lies in the slip or transition flow regimes. The mean free path for saturated water vapor at 2 psia is approximately $1 \mu \mathrm{m}$. Thus a $2-\mu \mathrm{m}$ water droplet would have a Knudsen number of
0.5. The effect of a Knudsen number of this magnitude on the droplet drag coefficient is discussed below.

The drag coefficient of a droplet is most strongly influenced by Reynolds number. If the Reynolds number is less than unity, the drag coefficient is given by Stokes law:

$$
C_{D}=\frac{24}{\operatorname{Re}}
$$

For Reynolds numbers of one or more, Stokes law underpredicts the drag coefficient as one can easily discern by looking at the standard drag coefficient curve for a sphere. An empirical equation for $C_{D}$ valid up to Reynolds number of 1000 is $^{11}$

$$
\frac{C_{D} \operatorname{Re}}{24}=1+.15 \operatorname{Re}^{0.687} \text {, }
$$

which, one notes, approaches Stokes law as the Reynolds number approaches zero. The expression is valid over the Reynolds number range encountered by droplets in geothermal calculations. Defining $f$ as

$$
f=\frac{C_{D} R e}{24} \text {, }
$$

one finds it is most convenient to write the aerodynamic drag force on a droplet as

$$
F_{\mu, 1}=3 \pi \mu d f\left(v_{1}-u_{1}\right)
$$

which again reduces to Stokes drag as $f$ approaches unity.

As an example of the magnitude of Reynolds number and drag coefficient to be expected, consider a 5- $\mu \mathrm{m}$ droplet in water vapor at 2 psia and assume a relative velocity of $100 \mathrm{ft} / \mathrm{sec}$. The corresponding Reynolds number of the droplet is 
approximately unity. Thus Stokes law is applicable and $f$ is also equal to unity. The question arises as to magnitude of rarefaction effects when the Knudsen number is 0.5 . Crowe ${ }^{12}$ has developed an empirical expression for the drag coefficient of a sphere for all Mach numbers and Reynolds numbers up to $10^{5}$. If the Reynolds number is unity or less, the factor $f$ is given

$$
f=1-0.05 \mathrm{Re} \mathrm{Kn} \text {, }
$$

so that a Knudsen number of 0.5 wi11 reduce f by approximately $2 \%$ which can be neglected.

In conclusion, the aerodynamic drag force on the droplet is well represented by the drag coefficient for a sphere at the appropriate Reynolds number.

\section{Buoyancy}

The buoyancy force on a droplet is due to a pressure gradient in the vapor phase in the same manner as the buoyancy force in a quiescent fluid is due to the pressure gradient due to gravity. The magnitude and direction of the force is

$$
F_{p, 1}=-v_{p} \frac{\partial p}{\partial x_{1}}
$$

where $v_{p}$ is the volume of the droplet.

The relative importance of the buoyant force can be assessed by comparing Its magnitude to the mass times acceleration of droplet:

$$
\frac{F_{p}}{m \alpha_{d}} \sim \frac{v_{p}}{\rho_{d} v_{p}} \frac{\frac{\partial p}{\partial x}}{\alpha_{d}}
$$

The pressure gradient in the vapor can be approximated by

$$
\frac{\partial p}{\partial x} \sim \rho_{v} \alpha
$$

$\mathbf{8 0}$

$$
\frac{F_{p}}{m \alpha_{d}} \sim \frac{\rho_{v}}{\rho_{d}} \frac{\alpha_{v}}{\alpha_{d}} .
$$

If the acceleration of the droplet and vapor are comparable, then the buoyant force/inertial force ratio is approximately equal to the density ratio which is generally $10^{-3}$. Under such conditions, the buoyancy force can be neglected. Thus the buoyancy force term may only be significant in regions of large vapor accelerations such such as in the throat region of a nozzle or through a shock wave.

Virtual Mass Effect

When a droplet accelerates with respect to the vapor, a force is developed on the droplet which is proportional to the relative acceleration. This force, called the virtual or apparent mass effect, is given by

$$
\begin{aligned}
F_{m, 1} & =k_{m} \rho_{v} v_{d}\left(\frac{d v_{i}}{d t}-\frac{d u_{i}}{d t}\right) \\
& =k \rho_{v} v_{d} \alpha_{r, i}
\end{aligned}
$$

where $K_{m}$ is an empirical constant. The virtual mass effect arises because of the acceleration of the surrounding vapor due to relative acceleration of the droplet. The acceleration of the vapor accrues from an additional force on the droplet. For a sphere, the theoretically derived value for $k$ is 0.5 , so the virtual mass effect can be thought of as the force necessary to accelerate a mass of vapor in a volume equal to half the volume displaced by the droplet. Further experiments by odar 13 
suggest the empirical constant depends on the acceleration modulus, $A c$, as

$$
K_{m}=1.05-\frac{0.066}{A c^{2}+0.12} \text {, }
$$

where Ac is defined as

$$
A c=\frac{\left|v_{1}-u_{i}\right|^{2}}{\alpha_{d} d}
$$

which is the ratio of dynamic' to acceleration-induced forces.

Comparing the virtual mass effect to the mass times acceleration of the droplet as done with the pressure gradient term, one arrives at the same conclusion pertaining to its significance.

\section{Basset Force}

Whereas the virtual mass effect is operative in a viscous or inviscid fluid, the Basset force only occurs in a viscous fluid and relates to the unsteadiness of the boundary layer. For example, consider a flat plate which is impulsively started in a quiescent fluid. As the momentum diffuses from the plate and a boundary layer develops, the shear stress produced by the boundary layer continually changes with time until the steady state condition is achieved. The deviation of the shear stress during the transient period from the steady-state value is the Basset force. Thus, the Basset force depends on the history of the relative acceleration and is given by

$$
F_{B, 1}=K_{B} d^{2}\left(\pi \rho_{v} \mu\right)^{1 / 2} \int_{0}^{t} \frac{\alpha_{r, 1}\left(t^{\prime}\right) d t^{\prime}}{\left(t-t^{\prime}\right)^{1 / 2}}
$$

Basset, by a theoretical calculation, 14 . showed that $K_{B}=6$. Experimental studies by $\operatorname{odar}^{13}$ indicate $\mathrm{K}_{B}$ depends on the acceleration modulus and is represented empirically by

$$
\mathrm{K}_{\mathrm{B}}=2.88+\frac{3.12}{(\mathrm{AC}+1)^{3}}
$$

Dividing the Basset force by the mass times acceleration of the droplet, one can show that

$$
\frac{F_{B}}{m \alpha_{d}} \curvearrowright 20\left(\frac{\rho_{v}}{\rho_{d}}\right)\left(\frac{A c}{R e}\right)^{1 / 2}
$$

assuming a constant relative acceleration. One notes that this term is generally small since the density ratio is of the order of $10^{-3}$ but may become significant in regions where Ac/Re is large, such as for small droplets in shock waves.

To summarize, the largest aerodynamic force acting on a droplet in the drag direction is the conventional aerodynamic drag with the other effects becoming significant only in regions of very high vapor acceleration.

\section{Magnus Effect}

The Magnus effect is the lift force due to rotation of the droplet and $1 \mathrm{~s}$ given by ${ }^{2}$

$F_{r, 1}=\varepsilon_{1 \alpha \beta} \frac{\pi}{8} \rho_{v} d^{3}\left(u_{\alpha}-v_{\alpha}\right) \omega_{\beta}$,

where $w_{f}$ is the rotational rate of the droplet. It is difficult to assess the rotational rate of the droplet especially if the droplet has rebounded from a wall. 
If the droplets are small and not rebounding from walls, the rotational rate is likely equal to that of the fluid, namely,

$$
\omega_{i}=\frac{\varepsilon_{1 \alpha \beta}}{2} \frac{\partial u_{\alpha}}{\partial x_{\beta}}
$$

\section{Saffman Lift Force}

The Saffman lift force is due to a velocity gradient in the fluid as shown in Fig. 6. The velocity gradient effects a higher velocity at $A$ than at $B$ and results In a lift force on the droplet. The droplet need not be rotating and ts thereby distinct from the Magnus effect. The Saffman $11 \mathrm{ft}$ force is given by ${ }^{2}$

$F_{s}=1.61\left(\mu \rho_{v}\right)^{1 / 2} d^{2}(v-u)\left|\frac{d v}{d y}\right|^{1 / 2},(108)$ which is valid only for low Reynolds ( $<1)$ numbers. There appear to be no data for the Saffman lift force at higher Reynolds numbers.

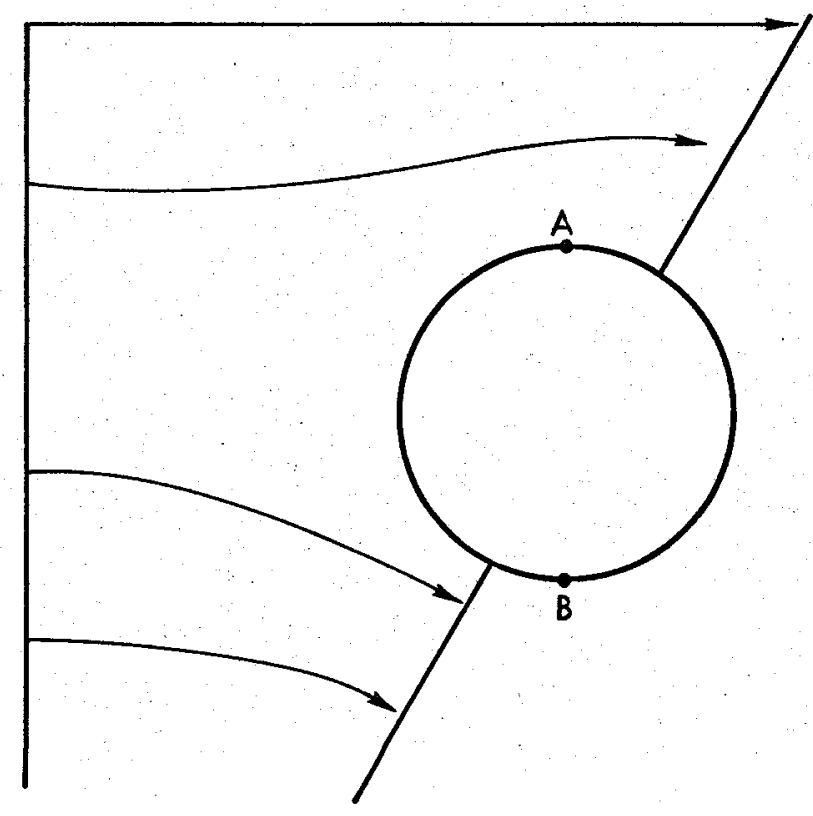

Fig. 6. Droplet in a shear flow.
Comparing the Saffman and Magnus forces on a droplet, assuming the droplet is rotating at the rotational rate of the fluid, one finds

$$
\frac{F_{s}}{F_{r}} \sim 11 \frac{u}{\rho_{v} u_{*} d}
$$

where $u_{*}$ is the shear velocity in the boundary layer. Assuming a 5- $\mu \mathrm{m}$ water droplet in saturated water vapor at 2 psia and a free stream velocity of $500 \mathrm{ft} / \mathrm{sec}$, one estimates

$$
\frac{\rho_{v} d u_{*}}{\mu} \sim 0.2
$$

so the Saffman lift force is an order of magnitude larger than the Magnus force. If, however, the droplet acquires a rotation different from that of the fluid, the Magnus force could become considerably larger.

\section{HEAT TRANSFER}

The principal mode of heat transfer between a droplet and vapor is forced convection. The temperatures associated with geothermal applications are too low to have any significant energy exchange by radiation. The rate of heat transfer by convection is quantified by the Nusselt number, defined by

$$
N u=\frac{\dot{Q}_{d}}{4 \pi d k\left(T-T_{d}\right)}
$$

where $k$ is the thermal conductivity and $\dot{Q}_{d}$ is the rate of heat transfer to the droplet. 
The Nusselt number depends on both the Reynolds number and the Prandt 1 number because both relate to the thickness of the thermal boundary layer and, hence, the temperature gradient. The suggested empirical correlation $1 s^{15}$

$$
\mathrm{Nu}=2+0.6 \mathrm{Re}^{1 / 2} \mathrm{Pr}^{1 / 3} \text {, }
$$

where Prandtl number is defined as

$$
\operatorname{Pr}=\frac{\mu}{K}
$$

and is the ratio of momentum to thermal diffusivity. The Prandtl number for steam is approximately 0.9 over a wide range of temperatures.

\section{Acknowledgments}

The author is indebted to Bill Comfort, who encouraged the preparation of this report. The author also acknowledges the stimulating discussions with B11l Comfort, Warren Gledt, and Terry Alger, many of the outcomes of which are included in this report. 


\section{References}

1. S. L. Soo, Fluid Dynamics of Multiphase Systems (Blalsdell Publishing Co., Waltham, Mass., 1967).

2. R. G. Boothroyd, Flowing Gas-Solids Suspensions (Chapman and Hall Ltd., London, 1971).

3. J. R. Kliegel, "Gas-Part1cle Nozzle Flows," N1nth Symposium (Internationa1) on Combustion (Academic Press, 1963).

4. D. Migdal, and V. D. Agosta, "A Source Flow Model for Continuous Gas-Particle Flow," J. Applied Mechanics 35 (4), 868 (1967).

5. J. R. Kllegel, One-Dimensional Flow of a Gas-Particle System, Institute of Aeronautical Sciences (now American Institute of Aeronautics and Astronautics), New York, Rept. 60-65 (1960).

6. J. A. Hultberg and S. L. Soo, "Two-Phase Flow Through a Nozzle," Astronautica Acta 11, 207 (1965).

7. D. A. Drew, "Averaged Fleld Equations for Two-Phase Media," Studies in Applied Math $\underline{L}$ (2) (1971).

8. C. Truesdell and R. Toupin, "The Classical Field Theories," in Handbuch der Physik (Springer Verlog, Berlin, 1960), Vol. 3/1.

9. H. S. Carslaw and J. C. Jaeger, Conduction of Heat in Solids (clarendon, Oxford, 1959).

10. G. H. Balley; I. V. Slater, and P. Elsenklam, "Dynamic Equations and Solutions for Particles Undergoing Mass Transfer," Brit. Chem Engr 15 (7), 912 (1970).

11. G. B. Wallis, One-Dimensional Two-Phase Flow (McGraw-Hill, New York, 1969).

12. C. T. Crowe et al., "Drag Coefficlent for Particles in Rarefied, Low Mach Number Flows," in Progress in Heat and Mass Transfer (Pergamon Press, New York, 1972), Vo1. 6.

13. F. Odar, "Verffication of the Proposed Equation for Calculation of Forces on a Sphere Accelerating in a Viscous Fluid," J. Fluid Mechanics 25 (3), 591 (1966).

14. A. B. Basset, A Treatise on Hydrodynamics (Defghton, Bell and Co., Cambridge, 1888), Vol. 2 .

15. R. B. Bird, W. E. Stewart, and E. N. Lightfoot, Transport Phenomena (Wiley, New York, 1960).

$\mathrm{MBB} / \mathrm{gw}$ 